\title{
LINEAR DYNAMICAL SYSTEMS OF NILPOTENT LIE GROUPS
}

\author{
INGRID BELTIŢĂ AND DANIEL BELTIŢĂ
}

\begin{abstract}
We study the topology of orbits of dynamical systems defined by finite-dimensional representations of nilpotent Lie groups. Thus, the following dichotomy is established: either the interior of the set of regular points is dense in the representation space, or the complement of the set of regular points is dense, and then the interior of that complement is either empty or dense in the representation space. The regular points are by definition the points whose orbits are locally compact in their relative topology. We thus generalize some results from the recent literature on linear actions of abelian Lie groups. As an application, we determine the generalized $a x+b$-groups whose $C^{*}$-algebras are antiliminary, that is, no closed 2-sided ideal is type I.

2010 MSC: Primary 22E27 Secondary 22E25, 22D25, 17B30

Keywords: semidirect product; group action; locally closed orbit; antiliminary locally compact group; Mautner group
\end{abstract}

\section{INTRODUCTION}

One of the main results of the present paper is that if $\pi: G \rightarrow \operatorname{End}(\mathcal{V})$ is a finite-dimensional representation of a nilpotent Lie group and $\Gamma$ is the set of all regular points $v \in \mathcal{V}$ (i.e., the corresponding orbit $\pi(G) v$ is locally compact in its relative topology), then either the interior of $\Gamma$ is dense in $\mathcal{V}$ or the complement $\mathcal{V} \backslash \Gamma$ is a dense subset of $\mathcal{V}$ whose interior is either empty or is dense in $\mathcal{V}$. (See Corollary 4.11,) When $G$ is an abelian Lie group, that dichotomy was essentially established in ACO16 and, as shown in Remark 4.15, it is closely related to a result of this type that had been obtained in [Pu71, Ch. IV, Prop. 8.2] for the coadjoint representation of any connected, simply connected, solvable Lie group $S$, and the latter result had been used for proving that almost all factors of the von Neumann algebra of $S$ are either type I or type II. In fact, one of the motivations of the present paper was to complete this picture by obtaining some information on the size of the set of unitary equivalence classes of the remaining, type III, factor representations. This, if $G$ is abelian, we show that either $\operatorname{Int} \Gamma$ is dense in $\mathcal{V}$ or the solvable Lie group $\mathcal{V}^{*} \rtimes G$ is antiliminary. (See Corollary 4.13, Remark [5.6] and Example 5.7)

To obtain our results we use some techniques from linear algebra, namely rationality properties of oblique projections and Moore-Penrose inverses, which allow us to improve the methods of investigation of the paper ACO16 mentioned above. From this perspective, this paper is a sequel to our earlier study from [BB17.

In Section 2 we establish some auxiliary results of two types: reduction theory for regular orbits of locally compact group actions, and weight space decompositions with respect to nilpotent Lie groups. In Section 3 we develop the linear algebra tools we need for the investigation of regular points. In Section 4 we establish the dichotomies for the sets of regular points in linear dynamical systems of general nilpotent Lie groups (Theorem 4.10 and Corollary 4.11). Finally, in Section 5 
we give an application to representation theory of some solvable Lie groups that may not be type I. Specifically, we give a precise characterization of the so-called generalized $a x+b$-groups whose $C^{*}$-algebra is antiliminary (Theorem 5.5).

Besides representation theory [ACD19, the study of regular points of dynamical systems holds an important role in several research areas, including for instance admissibility in continuous wavelet theory [ACDO13, BCFM15, ACO16, $C^{*}$ dynamical systems Wi07, [BB18.

General notation and terminology. We denote Lie groups by upper case Roman letters and their Lie algebras by the corresponding lower case Gothic letters. By a nilpotent Lie group we always understand a connected simply connected nilpotent Lie group.

For any subset of a real linear vector space $S \subset \mathcal{W}$, we denote by $\langle S\rangle$ the additive subgroup of $(\mathcal{W},+)$ generated by $S$.

For any finite-dimensional real vector space $\mathfrak{U}$, the Grassmann manifold of $\mathfrak{U}$ is the set $\operatorname{Gr}(\mathfrak{U})$ of all linear subspaces of $\mathfrak{U}$. When $\mathfrak{U}_{0} \subseteq \mathfrak{U}$ is linear subspace, we denote $\operatorname{Gr}_{\mathfrak{U}_{0}}(\mathfrak{U}):=\left\{\mathfrak{W} \in \operatorname{Gr}(\mathfrak{U}) \mid \mathfrak{U}_{0}+\mathfrak{W}=\mathfrak{U}\right\}$. (See [BB17] and [ACM13.)

For any complex Hilbert space $\mathcal{H}$ we denote by $\mathcal{B}(\mathcal{H})$ the von Neumann algebra of all bounded linear operators on $\mathcal{H}$, and by $\mathcal{K}(\mathcal{H})$ the set of all compact operators on $\mathcal{H}$.

For any real finite-dimensional Hilbert spaces $\mathcal{H}_{1}, \mathcal{H}_{2}$ we use again the notation $\mathcal{B}\left(\mathcal{H}_{1}, \mathcal{H}_{2}\right)$ for the space of the (bounded) $\mathbb{R}$-linear operators $\mathcal{H}_{1} \rightarrow \mathcal{H}_{2}$. When $\mathcal{V}$ is a $\mathbb{K}$ vector space with $\mathbb{K}=\mathbb{R}$ or $\mathbb{C}$, without considering any Hilbert space structure, we denote by $\operatorname{End}_{\mathbb{K}}(\mathcal{V})$ the space of $\mathbb{K}$-linear operators in $\mathcal{V}$. When $\mathbb{K}=\mathbb{R}$ and no confusion arises, we write simply $\operatorname{End}(\mathcal{V})$.

\section{Preliminaries}

In this section we collect two kinds of technical results for later use in this paper. Thus, we firstly develop a purely topological approach to the regularity of group orbits, based on the idea of reduction of symmetry groups that is used in other areas, for instance in geometric mechanics. To this end, we actually establish on their natural level of generality certain facts on Lie group actions that can be found in ACDO13. In the second part of this section we record some basic properties of weight-space decompositions of representations of nilpotent Lie algebras, a topic that is quite classical in Lie theory, for instance in the study of Cartan subalgebras. Here we just point out some features of the interaction between the weight-space decompositions and the operation of complexification. This topic was also discussed in ACDO13, in the special case of abelian Lie algebras.

\subsection{Reduction theory for regular orbits of locally compact group actions.}

Definition 2.1. For any fixed continuous action $G \times X \rightarrow X,(g, x) \mapsto g \cdot x$ of a locally compact group $G$ on a topological space $X$, a point $x_{0} \in X$ is called a regular point if its corresponding orbit $G . x_{0}$ is locally compact, and then G. $x_{0}$ is called a regular orbit. Here and everywhere in this paper, the orbits are endowed with their relative topology, that is, we regard the orbits as topological subspaces of the ambient space $X$.

One of the reason why the regular orbits are interesting is the following result of aHWi02, where we use the notation $\mathcal{C}_{0}(X)$ for the continuous functions that vanish at $\infty$ on a locally comact space $X$. 
Lemma 2.2. Let $\alpha: G \times X \rightarrow X$ be a continuous action of a locally compact group $H$ on a locally compact space $X$. Assume that both $G$ and $X$ are second countable and $H$ is amenable. If $H$ is abelian, then the largest type I ideal of the crossedproduct $C^{*}$-algebra $G \ltimes \mathcal{C}_{0}(X)$ is $G \ltimes \mathcal{C}_{0}\left(X_{0}\right)$, where $X_{0}$ is the set of all points $x \in X$ having a neighborhood $U_{x}$ for which the orbit of every point in $U_{x}$ is regular.

Proof. Since both $G$ and $X$ are second countable and $G$ is amenable, it follows that $G \ltimes \mathcal{C}_{0}(X)$ is EH-regular, as noted in aHWi02, page 537]. Then the assertion follows by aHWi02, Th. 3.16 and Rem. 3.17].

Remark 2.3. In the case when $X$ is actually a vector space $\mathcal{V}$ and $\alpha$ is an action by linear maps, we can form the semidirect product group $\mathcal{V} \rtimes_{\alpha} G$. Then there is a $*$-isomorphism $C^{*}\left(\mathcal{V} \rtimes_{\alpha} G\right) \simeq G \ltimes_{\alpha^{*}} \mathcal{C}_{0}\left(\mathcal{V}^{*}\right)$, by [Wi07, Ex. 3.16], where $\alpha^{*}: G \times \mathcal{C}_{0}\left(\mathcal{V}^{*}\right) \rightarrow \mathcal{C}_{0}\left(\mathcal{V}^{*}\right), \alpha^{*}(g, f):=f \circ \alpha_{g^{-1}}$.

The next lemma is a generalization of ACDO13, Cor. 2.3] from Lie group actions to locally compact group actions.

Lemma 2.4. Let $G \times X \rightarrow X,(g, x) \mapsto g \cdot x$, be a continuous action of a second countable, locally compact group on a Hausdorff topological space. For any $x_{0} \in X$ with its orbit $\mathcal{O}:=G . x_{0}$, then the following assertions are equivalent:

(i) The orbit $\mathcal{O}$ is regular.

(ii) For every compact neighborhood $K$ of $\mathbf{1} \in G$ there exists a neighborhood $V$ of $x_{0} \in X$ with $V \cap \mathcal{O} \subseteq K . x_{0}$.

(iii) There exist a compact neighborhood $K$ of $\mathbf{1} \in G$ and a neighborhood $V$ of $x_{0} \in X$ with $V \cap \mathcal{O} \subseteq K . x_{0}$.

If the space $X$ is first countable, the group $G$ is noncompact and countable at infinity, and the mapping $G \rightarrow X, g \mapsto g \cdot x_{0}$, is injective, then the above assertions are equivalent to the following one:

(iv) There is no sequence $\left\{g_{n}\right\}_{n \in \mathbb{N}}$ in $G$ satisfying $\lim _{n \in \mathbb{N}} g_{n}=\infty$ and $\lim _{n \in \mathbb{N}} g_{n} . x_{0}=x_{0}$.

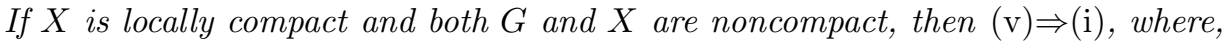

(v) One has $\lim _{g \rightarrow \infty} g \cdot x_{0}=\infty$ in $X$.

Proof. (ii) $\Rightarrow$ (iii) Let $L$ be any compact neighborhood of $\mathbf{1} \in G$ with $L^{-1} L \subseteq K$. One has $G=\bigcup_{g \in G} g L$ hence, since $G$ is second countable, it has a subset $C \subseteq G$ that is at most countable and satisfies $G=\bigcup_{c \in C} c L$. Since $\mathcal{O}=G \cdot x_{0}$, we then obtain $\mathcal{O}=\bigcup_{c \in C} c L . x_{0}$. This is a countable union of compact subsets of $\mathcal{O}$. Since $\mathcal{O}$ is locally compact by hypothesis, it then follows by Baire's theorem that there exists $c \in C$ for which the set $c L . x_{0} \subseteq \mathcal{O}$ has nonempty interior. Using the homeomorphism $\mathcal{O} \rightarrow \mathcal{O}, x \mapsto c^{-1} . x$, we then obtain that the interior of $L . x_{0}$ is nonempty, hence there exists $g \in L$ for which $g . x_{0}$ belongs to the interior of $L . x_{0}$. Then $x_{0}$ belongs to the interior of $g^{-1} L . x_{0}$. On the other hand $g^{-1} L . x_{0} \subseteq L^{-1} L . x_{0} \subseteq K . x_{0}$, hence $x_{0}$ belongs to the interior of $K \cdot x_{0}$, which is equivalent to Assertion (iii).

(iii) $\Rightarrow$ (iii) Obvious.

(iiii) $\Rightarrow$ (ii) It follows by (iii) that $K \cdot x_{0}$ is a compact neighborhood of $x_{0} \in \mathcal{O}$. Since the group $G$ acts transitively on $\mathcal{O}$ by homeomorphisms, it then follows that every point of $\mathcal{O}$ has a compact neighborhood, hence $\mathcal{O}$ is locally compact. 
Now assume that $G$ is noncompact and countable at infinity, hence it has a sequence of compact subsets $K_{1} \subseteq K_{2} \subseteq \cdots \subseteq G$ with $\bigcup_{n \geq 1} K_{n}=G$. We also assume that $X$ is first countable and the mapping $G \rightarrow X, g \mapsto g . x_{0}$, is injective.

(iii) $\Rightarrow$ (iv) Let $\left\{g_{n}\right\}_{n \in \mathbb{N}}$ be a sequence in $G$ with $\lim _{n \in \mathbb{N}} g_{n}=\infty$ and $\lim _{n \in \mathbb{N}} g_{n} . x_{0}=x_{0}$. Using the notation of (iii), there exists $n_{V} \in \mathbb{N}$ with $g_{n} . x_{0} \in V \cap \mathcal{O} \subseteq K . x_{0}$ for every $n \geq n_{V}$. Since the mapping $G \rightarrow X, g \mapsto g . x_{0}$ is injective, we then obtain $g_{n} \in K$ for every $n \geq n_{V}$. Since the group $G$ is noncompact, this contradicts the assumption $\lim _{n \in \mathbb{N}} g_{n}=\infty$.

(iv) $\Rightarrow$ (ii) Reasoning by contradiction, we assume that there exists a compact neighborhood $K$ of $\mathbf{1} \in G$ such that for every neighborhood $V$ of $x_{0} \in X$ one has $V \cap \mathcal{O} \nsubseteq K . x_{0}$. Since $X$ is first countable, there exists a countable base of neighborhoods $\left\{V_{n}\right\}_{n \in \mathbb{N}}$ of $x_{0} \in X$. Then for every $n \in \mathbb{N}$ one has by assumption $V_{n} \cap \mathcal{O} \nsubseteq K . x_{0}$, hence there exists $y_{n} \in V_{n} \cap \mathcal{O}$ with $y_{n} \notin K . x_{0}$. Since $y_{n} \in \mathcal{O}$ and the mapping $G \rightarrow X, g \mapsto g \cdot x_{0}$, is injective, there exists a unique element $g_{n} \in G$ with $y_{n}=g_{n} . x_{0}$. Moreover, since $y_{n} \in V_{n}$ for all $n \in \mathbb{N}$, we obtain $\lim _{n \in \mathbb{N}} g_{n} . x_{0}=x_{0}$.

It then follows by the hypothesis (iv) that one does not have $\lim _{n \in \mathbb{N}} g_{n}=\infty$. Then, selecting a suitable subsequence, one may assume that there exists $h \in G$ with $\lim _{n \in \mathbb{N}} g_{n}=h$, hence $h \cdot x_{0}=\lim _{n \in \mathbb{N}} g_{n} . x_{0}=x_{0}$ Since the mapping $G \rightarrow X, g \mapsto g \cdot x_{0}$, is injective, it then follows that $h=\mathbf{1} \in G$, hence $\lim _{n \in \mathbb{N}} g_{n}=\mathbf{1}$. On the other hand $g_{n} . x_{0}=y_{n} \notin K . x_{0}$ hence, by the injectivity of the mapping $g \mapsto g . x_{0}$ again, we obtain $g_{n} \notin K$ for all $n \in \mathbb{N}$. Since $K$ is a neighborhood of $\mathbf{1} \in G$, we thus obtain a contradiction.

We now assume that the space $X$ is locally compact and noncompact.

(V) $\Rightarrow$ (ii) Denote by $\widehat{G}=G \sqcup\left\{\infty_{G}\right\}$ and $\widehat{X}=X \sqcup\left\{\infty_{X}\right\}$ the one-point compactifications of $G$ and $X$, respectively, and define the continuous mapping $\psi: G \rightarrow X$, $\psi(g):=g \cdot x_{0}$. It follows by the hypothesis ( $\left.\mathbf{\nabla}\right)$ that $\psi$ extends by continuity to a continuous mapping $\widehat{\psi}: \widehat{G} \rightarrow \widehat{X}$ with $\widehat{\psi}\left(\infty_{G}\right)=\infty_{X}$. Since $\widehat{G}$ is compact, it follows that $\widehat{\psi}(\widehat{G})$ is a compact subset of $\widehat{X}$. Then $\widehat{\psi}(\widehat{G}) \backslash\left\{\infty_{X}\right\}$ is a locally closed subset of $\widehat{X}$. Since $\widehat{\psi}(\widehat{G}) \backslash\left\{\infty_{X}\right\} \subseteq X$ and $X$ is an open subset of $\widehat{X}$, it also follows that $\widehat{\psi}(\widehat{G}) \backslash\left\{\infty_{X}\right\}$ is a locally closed subset of $X$. Since $\widehat{\psi}(\widehat{G}) \backslash\left\{\infty_{X}\right\}=\psi(G)=G \cdot x_{0}=\mathcal{O}$, we thus see that $\mathcal{O}$ is a locally cosed subset of $X$, and we are done.

The following lemma is a generalization of [ACDO13, Prop. 3.1] from Lie group actions to locally compact group actions. For a continous action $G \times X \rightarrow X$, $(g, x) \mapsto g . x$ of $G$ in a topological space $X$, and $x \in X$, we denote by $G(x)$ the corresponding isotropy group, that is, $G(v):=\{g \in G \mid g \cdot x=x\}$.

Lemma 2.5. Let $G \times X \rightarrow X$ and $G \times W \rightarrow W$ be continuous actions of a second countable, locally compact group on Hausdorff topological spaces and assume that $q: X \rightarrow W$ is a continuous surjective mapping which is $G$-equivariant. For any $x_{0} \in X$ we denote $w_{0}:=q\left(x_{0}\right), \mathcal{O}:=G . x_{0}, \mathcal{O}_{0}:=G\left(w_{0}\right) . x_{0}$, and $\mathcal{O}_{w_{0}}:=G . w_{0}$. Then the following assertions hold:

(i) One has $\mathcal{O} \cap q^{-1}\left(w_{0}\right)=\mathcal{O}_{0}$ and $q(\mathcal{O})=\mathcal{O}_{w_{0}}$.

(ii) If $\mathcal{O}$ is a locally compact subset of $X$, then $\mathcal{O}_{0}$ is a locally compact subset of $X$. 
(iii) If $\mathcal{O}_{0}$ is a locally compact subset of $X$ and $\mathcal{O}_{w_{0}}$ is a locally compact subset of $W$, then $\mathcal{O}$ is a locally compact subset of $X$.

(iv) If $\mathcal{O}_{w_{0}}$ is a locally compact subset of $W$, then one has

$$
\mathcal{O} \text { locally compact } \subseteq X \Longleftrightarrow \mathcal{O}_{0} \text { locally compact } \subseteq X .
$$

Proof. (ii) This is straightforward.

(ii) This follows by Assertion (ii) since the intersection of a compact set with a closed set is compact.

(iii) Let $K$ be an arbitrary compact neighborhood of $1 \in G$, and select any compact neighborhood $K_{1}$ of $\mathbf{1} \in G$ with $K_{1}$ contained in the interior of $K$.

It follows by the hypothesis that $\mathcal{O}_{0}$ is locally compact along with Lemma 2.4 and Assertion (iii) that there exist a neighborhood $V_{1}$ of $x_{0} \in X$ satisfying

$$
V_{1} \cap \mathcal{O} \cap q^{-1}\left(w_{0}\right) \subseteq K_{1} \cdot x_{0}
$$

Using the continuity of the group action $G \times X \rightarrow X$ at $\left(\mathbf{1}, x_{0}\right) \in G \times X$ we now select a compact neighborhood $K_{0}$ of $\mathbf{1} \in G$ and a neighborhood $V_{2}$ of $x_{0} \in X$ with

$$
K_{0}^{-1} \cdot V_{2} \subseteq V_{1} \text {. }
$$

Since $K_{1}$ is a compact contained in the interior of $K$ we may shrink $K_{0}$ in order to have

$$
K_{0} K_{1} \subseteq K .
$$

Using the hypothesis that $\mathcal{O}_{w_{0}}$ is locally compact in $W$, we obtain by Lemma 2.4 again a neighborhood $V_{w_{0}}$ of $w_{0} \in W$ with

$$
V_{w_{0}} \cap \mathcal{O}_{w_{0}} \subseteq K_{0} \cdot w_{0} .
$$

We now prove that $V:=V_{2} \cap q^{-1}\left(V_{w_{0}}\right)$ is a neighborhood of $x \in X$ satisfying

$$
V \cap \mathcal{O} \subseteq K \cdot x_{0}
$$

and then $\mathcal{O}$ is locally closed by Lemma 2.4 .

It is easily seen that $V$ is a neighborhood of $x \in X$. To prove the above inclusion, let $x \in V \cap \mathcal{O}$ arbitrary. Then one has $q(x) \in q\left(q^{-1}\left(V_{w_{0}}\right)\right)=V_{w_{0}}$ since $q$ is surjective, and on the other hand $q(x) \in q(\mathcal{O})=\mathcal{O}_{w_{0}}$ by Assertion (ii), hence $q(x) \in V_{w_{0}} \cap \mathcal{O}_{w_{0}} \subseteq K_{0} . w_{0}$. Thus there exists $k_{0} \in K_{0}$ with $q(x)=k_{0} . w_{0}$, and then $k_{0}^{-1} . x \in q^{-1}\left(w_{0}\right)$. Since $x \in \mathcal{O}$, we also obtain $k_{0}^{-1} \cdot x \in \mathcal{O}$. Moreover, since $x \in V \subseteq V_{2}$, we obtain

$$
k_{0}^{-1} \cdot x \in q^{-1}\left(w_{0}\right) \cap \mathcal{O} \cap K_{0}^{-1} \cdot V_{2} \subseteq q^{-1}\left(w_{0}\right) \cap \mathcal{O} \cap V_{1} \subseteq K_{1} \cdot x_{0}
$$

hence $x \in K_{0} K_{1} \cdot x_{0} \subseteq K . x_{0}$.

(iv) This directly follows by Assertions (iii)-(iii).

\subsection{Weight space decompositions with respect to nilpotent Lie algebras.}

Notation 2.6. We now introduce some notation to be used in the following, unless otherwise specified.

- $\mathcal{V}$ is a finite-dimensional real vector space with $m:=\operatorname{dim} \mathcal{V}$;

- $\mathfrak{g}$ is a nilpotent Lie algebra with its corresponding Lie group $G=(\mathfrak{g}, \cdot)$, where $\cdot$ is given by the Baker-Campbell-Hausdorff formula;

- $\mathfrak{g}_{\mathbb{C}}:=\mathbb{C} \otimes_{\mathbb{R}} \mathfrak{g}=\mathfrak{g} \dot{+} \mathfrak{i g}$ is the complexification of $\mathfrak{g}$, with its involutive antilinear map $\mathfrak{g}_{\mathbb{C}} \rightarrow \mathfrak{g}_{\mathbb{C}}, x \mapsto \bar{x}$ satisfying $\bar{x}=x$ for all $x \in \mathfrak{g}$; 
- the linear dual space of the complex Lie algebra $\mathfrak{g}_{\mathbb{C}}$ is denoted by $\mathfrak{g}_{\mathbb{C}}^{*}$, with its involutive antilinear map $\mathfrak{g}_{\mathbb{C}}^{*} \rightarrow \mathfrak{g}_{\mathbb{C}}^{*}, \lambda \mapsto \bar{\lambda}$, where $\bar{\lambda}(x):=\overline{\lambda(\bar{x})}$ for all $x \in \mathfrak{g}_{\mathbb{C}}$ and $\lambda \in \mathfrak{g}_{\mathbb{C}}^{*}$

- $\rho: \mathfrak{g} \rightarrow \operatorname{End}(\mathcal{V})$ is a Lie algebra representation, with its corresponding extension to a morphism of complex Lie algebras $\rho: \mathfrak{g}_{\mathbb{C}} \rightarrow \operatorname{End}_{\mathbb{C}}\left(\mathcal{V}_{\mathbb{C}}\right)$

- If $\mathcal{V}_{\mathbb{C}}:=\mathbb{C} \otimes_{\mathbb{R}} \mathcal{V}$ denotes the complexification of $\mathcal{V}$, then $C: \mathcal{V}_{\mathbb{C}} \rightarrow \mathcal{V}_{\mathbb{C}}$ is the involutive antilinear map satisfying $C v=v$ for all $v \in \mathcal{V}$.

Remark 2.7. For every $x \in \mathfrak{g}_{\mathbb{C}}, C \rho(x) C=\rho(\bar{x})$. Indeed, let $x_{1}, x_{2} \in \mathfrak{g}$ with $x=x_{1}+\mathrm{i} x_{2}$. Then $C \rho(x) C=C\left(\rho\left(x_{1}\right)+\mathrm{i} \rho\left(x_{2}\right)\right) C=\rho\left(x_{1}\right)-\mathrm{i} \rho\left(x_{2}\right)=\rho(\bar{x})$.

Lemma 2.8. For every $\lambda \in \mathfrak{g}_{\mathbb{C}}^{*}$, the set

$$
\mathcal{V}_{\mathbb{C}}^{\lambda}:=\bigcap_{x \in \mathfrak{g}_{\mathbb{C}}} \operatorname{Ker}(\rho(x)-\lambda(x) \mathrm{id})^{m} \subseteq \mathcal{V}_{\mathbb{C}}
$$

is an invariant subspace for the representation $\rho: \mathfrak{g}_{\mathbb{C}} \rightarrow \operatorname{End}_{\mathbb{C}}\left(\mathcal{V}_{\mathbb{C}}\right)$, and one has

$$
\mathcal{V}_{\mathbb{C}}=\bigoplus_{\lambda \in \Lambda} \mathcal{V}_{\mathbb{C}}^{\lambda}
$$

where the set $\Lambda:=\left\{\lambda \in \mathfrak{g}_{\mathbb{C}}^{*} \mid \mathcal{V}_{\mathbb{C}}^{\lambda} \neq\{0\}\right\}$ is finite and for every $\lambda \in \Lambda$ one has $\left[\mathfrak{g}_{\mathbb{C}}, \mathfrak{g}_{\mathbb{C}}\right] \subseteq \operatorname{Ker} \lambda$.

Proof. Since $\mathfrak{g}_{\mathbb{C}}$ is a complex nilpotent Lie algebra, the assertions follow for instance by [Ca05, Th. 2.9].

Lemma 2.9. For all $\lambda \in \mathfrak{g}_{\mathbb{C}}^{*}$ one has $C\left(\mathcal{V}_{\mathbb{C}}^{\lambda}\right)=\mathcal{V}_{\mathbb{C}}^{\bar{\lambda}}$.

Proof. For every $v \in \mathcal{V}_{\mathbb{C}}^{\lambda}$ and $x \in \mathfrak{g}_{\mathbb{C}}$ one has $(\rho(x)-\lambda(x) \mathrm{id})^{m} v=0$ hence, since $C^{2}=\mathrm{id}$,

$$
0=C(\rho(x)-\lambda(x) \mathrm{id})^{m} v=(C(\rho(x)-\lambda(x) \mathrm{id}) C)^{m} C v=(C \rho(x) C-\overline{\lambda(x)} \mathrm{id})^{m} C v
$$

By Remark 2.7 $C \rho(x) C=\rho(\bar{x})$, and thus the above equality implies

$$
\left(\forall x \in \mathfrak{g}_{\mathbb{C}}\right) \quad(\rho(\bar{x})-\overline{\lambda(x)} \mathrm{id})^{m} C v=0
$$

hence

$$
\left(\forall x \in \mathfrak{g}_{\mathbb{C}}\right) \quad(\rho(x)-\overline{\lambda(\bar{x})} \mathrm{id})^{m} C v=0
$$

Therefore $C v \in \mathcal{V}_{\mathbb{C}}^{\bar{\lambda}}$. Thus $C\left(\mathcal{V}_{\mathbb{C}}^{\lambda}\right) \subseteq \mathcal{V}_{\mathbb{C}}^{\bar{\lambda}}$, and the converse inclusion follows by symmetry, since $C^{2}=$ id. This completes the proof.

Definition 2.10. For any subset $\Phi \subseteq \Lambda$ we denote $\mathcal{V}_{\mathbb{C}}^{\Phi}:=\bigoplus_{\lambda \in \Phi} \mathcal{V}_{\mathbb{C}}^{\lambda}$ and we define the idempotent mapping $P_{\Phi} \in \operatorname{End}_{\mathbb{C}}(\mathcal{V})$ with $\operatorname{Ran} P_{\Phi}=\mathcal{V}_{\mathbb{C}}^{\Phi}$ and $\operatorname{Ker} P_{\Phi}=\mathcal{V}_{\mathbb{C}}^{\Lambda \backslash \Phi}$, using Lemma 2.8. If one has $\Phi=\{\lambda\}$ for some $\lambda \in \Lambda$, then we define $P_{\lambda}:=P_{\Phi}=P_{\{\lambda\}}$.

We denote $\Lambda^{\prime}:=\{\lambda \in \Lambda \mid \bar{\lambda}=\lambda\}$ and, using Lemma 2.9. we fix a subset $\Lambda^{\prime \prime} \subseteq \Lambda \backslash \Lambda^{\prime}$ satisfying $\Lambda \backslash \Lambda^{\prime}=\Lambda^{\prime \prime} \sqcup \overline{\Lambda^{\prime \prime}}$, where $\overline{\Lambda^{\prime \prime}}:=\left\{\bar{\lambda} \mid \lambda \in \Lambda^{\prime \prime}\right\}$. We then obtain the partition $\Lambda=\Lambda^{\prime} \sqcup \Lambda^{\prime \prime} \sqcup \overline{\Lambda^{\prime \prime}}$. This leads to the direct sum decomposition

$$
\mathcal{V}_{\mathbb{C}}=\mathcal{V}_{\mathbb{C}}^{\Lambda^{\prime}}+\mathcal{V}_{\mathbb{C}}^{\Lambda^{\prime \prime}}+\mathcal{V}_{\mathbb{C}}^{\overline{\Lambda^{\prime \prime}}}
$$

via Lemma 2.8 again. 
Lemma 2.11. The mapping

$$
\iota: \mathcal{V} \rightarrow\left(\mathcal{V} \cap \mathcal{V}_{\mathbb{C}}^{\Lambda^{\prime}}\right)+\mathcal{V}_{\mathbb{C}}^{\Lambda^{\prime \prime}}, \quad \iota(v):=P_{\Lambda^{\prime}} v+P_{\Lambda^{\prime \prime}} v
$$

is an $\mathbb{R}$-linear isomorphism.

Proof. One can easily check that $\operatorname{dim}_{\mathbb{R}} \mathcal{V}=\operatorname{dim}_{\mathbb{R}}\left(\left(\mathcal{V} \cap \mathcal{V}_{\mathbb{C}}^{\Lambda^{\prime}}\right) \dot{+} \mathcal{V}_{\mathbb{C}}^{\Lambda^{\prime \prime}}\right)$ and $\operatorname{Ker} \iota=\{0\}$, using Lemmas 2.9 and 2.8 .

Definition 2.12. We define the real vector space

$$
\widetilde{\mathcal{V}}:=\left(\mathcal{V} \cap \mathcal{V}_{\mathbb{C}}^{\Lambda^{\prime}}\right)+\mathcal{V}_{\mathbb{C}}^{\Lambda^{\prime \prime}}
$$

and the Lie algebra representation

$$
\widetilde{\rho}: \mathfrak{g} \rightarrow \operatorname{End}(\widetilde{\mathcal{V}}), \quad \widetilde{\rho}(x):=\iota \circ \rho(x) \circ \iota^{-1},
$$

using Lemma 2.11.

\section{Dichotomies For RATIONAL FUnCtions AND OBLIQUE PROJECTIONS}

In this section we obtain some auxiliary results that are needed in Section 4

3.1. Dichotomies for rational functions. The aim of this subsection is to obtain a version (Lemma 3.6) of some well-known results (see Remark 3.1), concerning finite families of rational $\mathcal{Y}$-valued functions rather than vectors of the finite-dimensional real vector space $\mathcal{Y}$.

Remark 3.1. Let $F \subseteq \mathcal{Y}$ be a finite subset of a finite-dimensional real vector space. Then the countable subgroup $\langle F\rangle=\sum_{f \in F} \mathbb{Z} f$ of $(\mathcal{Y},+)$ generated by $F$ is locally closed if and only if $\langle F\rangle$ is closed, and if and only if $\langle F\rangle$ is discrete.

In fact, every locally closed subgroup of a topological group is closed. Furthermore, if $\langle F\rangle$ is closed in $\mathcal{Y}$ and $\langle F\rangle$ is not discrete, then by Bo74, Ch. 5, $\S 1$, no. 1 , Cor.] there exists $y \in \mathcal{Y}$ with $\mathbb{R} y \subseteq\langle F\rangle$. This implies that $\langle F\rangle$ is not countable, which is a contradiction.

Lemma 3.2. Let $\mathcal{Y}$ be a finite-dimensional real vector space and $y_{1}, \ldots, y_{k} \in \mathcal{Y}$ be linearly independent vectors. For any positive integer $r \geq 1$ and any real numbers $a_{i j}$ for $1 \leq i \leq r$ and $1 \leq j \leq k$ define $z_{i}:=\sum_{j=1}^{k} a_{i j} y_{j} \in \mathcal{Y}$. Then the subgroup $\mathbb{Z} y_{1}+\cdots+\mathbb{Z} y_{k}+\mathbb{Z} z_{1}+\cdots+\mathbb{Z} z_{r}$ of $(\mathcal{Y},+)$ generated by $y_{1}, \ldots, y_{k}, z_{1}, \ldots, z_{r}$ is discrete/closed/locally closed if and only if for all $1 \leq i \leq r$ and $1 \leq j \leq k$ one has $a_{i j} \in \mathbb{Q}$.

Proof. Using Remark 3.1 the case $r=1$ follows by [Bo74, Ch. 5, §1, no. 1, Cor.]. The general case can then be proved by iteration, using the fact that any subgroup of a discrete group is discrete.

Definition 3.3. For any finite-dimensional real vector space $\mathcal{X}$ and any subset $U \subseteq \mathcal{X}$ we say that a function $f: U \rightarrow \mathbb{R}$ is rational if there exist polynomial functions $P, Q: \mathcal{X} \rightarrow \mathbb{R}$ satisfying $Q(x) \neq 0$ and $f(x)=P(x) / Q(x)$ for all $x \in U$. If $\mathcal{Y}$ is another finite-dimensional real vector space, then a vector function $\phi: U \rightarrow \mathcal{Y}$ is rational if for every linear functional $\xi: \mathcal{Y} \rightarrow \mathbb{R}$ the scalar function $\xi \circ \varphi: U \rightarrow \mathbb{R}$ is rational in the above sense. 
Lemma 3.4. Let $\mathcal{X}$ and $\mathcal{Y}$ be finite-dimensional real vector spaces, and fix any countable subset $S \subseteq \mathcal{Y}$. If $U \subseteq \mathcal{X}$ is an open subset and $f: U \rightarrow \mathcal{Y}$ is a rational function, then one of the following assertions holds:

(i) There exists $s \in S$ with $f(U)=\{s\}$.

(ii) The subset $f^{-1}(\mathcal{Y} \backslash S) \subseteq U$ is dense and, if moreover $\mathcal{Y} \backslash S$ is totally disconnected, then either $\operatorname{Int} f^{-1}(\mathcal{Y} \backslash S)=\emptyset$ or there exists $y \in \mathcal{Y} \backslash S$ with $f(U)=\{y\}$.

Proof. The assertions (ii) and (iii) cannot hold true simultaneously. Therefore it suffices to prove that if (ii) fails, then (iii) is true.

For every $s \in S$ the set $f^{-1}(s)$ is closed and, since $f$ is rational, we have that either Int $f^{-1}(s)=\emptyset$ or $f^{-1}(s)=U$. If the first assertion in the statement fails to be true, it then follows that for every $s \in S$ the subset $f^{-1}(s) \subseteq U$ is closed and has empty interior. Then, by Baire's category theorem the set $\bigcup_{s \in S} f^{-1}(s)$ has empty interior in $U$, that is, its complement is dense in $U$. One has $U \backslash \bigcup_{s \in S} f^{-1}(s)=$ $f^{-1}(\mathcal{Y} \backslash S)$, hence $f^{-1}(\mathcal{Y} \backslash S)$ is a dense subset of $U$.

Now assume that moreover $\mathcal{Y} \backslash S$ is totally disconnected and $\operatorname{Int} f^{-1}(\mathcal{Y} \backslash S) \neq \emptyset$. Then there exists an open connected nonempty subset $B \subseteq U$ with $f(B) \subseteq \mathcal{Y} \backslash S$. Since $B$ is connected and the function $f$ is continuous, the set $f(B)$ is in turn connected. The hypothesis that $\mathcal{Y} \backslash S$ is totally disconnected then implies that there exists $y_{0} \in \mathcal{Y} \backslash S$ with $f(B)=\left\{y_{0}\right\}$. Since the set $B$ is open and nonempty, and the function $f$ is rational, it then follows that $f$ is constant, hence $f(U)=\left\{y_{0}\right\}$. This completes the proof.

Lemma 3.5. Let $\mathcal{X}$ be a finite-dimensional real vector space and $U \subseteq \mathcal{X}$ be an open subset. Let $1 \leq k \leq m$ be any positive integers and assume that $y_{j}: U \rightarrow \mathbb{R}^{m}$ and $z_{i}: U \rightarrow \mathbb{R}^{m}$ are rational functions for $j=1, \ldots, k$ and $i=1, \ldots, r$, satisfying the conditions

$$
y_{1}\left(x_{0}\right), \ldots, y_{k}\left(x_{0}\right) \in \mathbb{R}^{m} \text { are linearly independent at some point } x_{0} \in U
$$

and

$$
z_{1}(x), \ldots, z_{r}(x) \in \operatorname{span}_{\mathbb{R}}\left\{y_{1}(x), \ldots, y_{k}(x)\right\} \text { for all } x \in U .
$$

If we denote

$$
A:=\left\{x \in U \mid z_{1}(x), \ldots, z_{r}(x) \in \operatorname{span}_{\mathbb{Q}}\left\{y_{1}(x), \ldots, y_{k}(x)\right\}\right\}
$$

then there exists a dense open subset $U_{0} \subseteq U$ for which exactly one of the following assertions holds:

(i) One has $U_{0} \subseteq A$ and there exist $a_{i j} \in \mathbb{Q}$ for $1 \leq i \leq r$ and $1 \leq j \leq k$ with $z_{i}(x)=\sum_{j=1}^{k} a_{i j} y_{j}(x)$ for $i=1, \ldots, r$ and all $x \in U_{0}$.

(ii) The set $U \backslash A$ is dense in $U$, and either $\operatorname{Int}(U \backslash A)=\emptyset$ or there exist $a_{i j} \in \mathbb{R}$ for $1 \leq i \leq r$ and $1 \leq j \leq k$ with $z_{i}(x)=\sum_{j=1}^{k} a_{i j} y_{j}(x)$ for $i=1, \ldots, r$ and all $x \in U_{0}$.

Proof. Assume $U \neq \emptyset$ and write

$$
y_{j}=\left(y_{j 1}, \ldots, y_{j m}\right): U \rightarrow \mathbb{R}^{m} \text { and } z_{i}=\left(z_{i 1}, \ldots, z_{i m}\right): U \rightarrow \mathbb{R}^{m}
$$


for $j=1, \ldots, k$ and $i=1, \ldots, r$. Since the vectors $y_{1}\left(x_{0}\right), \ldots, y_{k}\left(x_{0}\right) \in \mathbb{R}^{m}$ are linearly independent at some point $x_{0} \in U$, there exists a set $J=\left\{t_{1}, \ldots, t_{k}\right\} \subseteq$ $\{1, \ldots, m\}$ with $1 \leq t_{1}<\cdots<t_{k} \leq m$ and $x_{0} \in U_{J}$, where $U_{J}:=\left\{x \in U \mid \delta_{J}(x) \neq\right.$ $0\}$ and

$$
\delta_{J}: U \rightarrow \mathbb{R}, \quad \delta_{J}(x):=\operatorname{det}\left(y_{s, t_{j}}(x)\right)_{1 \leq s, j \leq k} .
$$

It is clear that $\delta_{J}: U \rightarrow \mathbb{R}$ is a rational function hence the set $U_{J}$ is a dense open subset of $U$.

Moreover, the vectors $y_{1}(x), \ldots, y_{k}(x) \in \mathbb{R}^{m}$ are linearly independent for every $x \in U_{J}$. It then follows by the hypothesis that for $i=1, \ldots, r$ and every $x \in U_{J}$ there exist uniquely determined $a_{i 1}(x), \ldots, a_{i k}(x) \in \mathbb{R}$ with $z_{i}(x)=\sum_{j=1}^{k} a_{i j}(x) y_{j}(x)$, that is,

$$
\left\{\begin{array}{l}
z_{i 1}(x)=a_{i 1}(x) y_{11}(x)+a_{i 2}(x) y_{21}(x)+\cdots+a_{i k}(x) y_{k 1}(x), \\
\cdots \ldots \ldots \\
z_{i m}(x)=a_{i 1}(x) y_{1 m}(x)+a_{i 2}(x) y_{2 m}(x)+\cdots+a_{i k}(x) y_{k m}(x) .
\end{array}\right.
$$

If $B_{J}(x):=\left(y_{s, t_{j}}(x)\right)_{1 \leq s, j \leq k} \in M_{k}(\mathbb{R})$, then $\operatorname{det} B_{J}(x)=\delta_{J}(x) \neq 0$ for all $x \in U_{J}$, hence the above overdetermined linear system can be solved for $a_{i 1}(x), \ldots, a_{i k}(x)$ by

$$
\underbrace{\left(z_{i, t_{1}}(x) \ldots z_{i, t_{k}}(x)\right)}_{\in M_{1, k}(\mathbb{R})} \underbrace{B_{J}(x)^{-1}}_{\in M_{k}(\mathbb{R})}=\underbrace{\left(a_{i 1}(x) \ldots a_{i k}(x)\right)}_{\in M_{1, k}(\mathbb{R})}
$$

This shows that $a_{i 1}, \ldots, a_{i k}$ are rational functions on $U_{J}$ for $i=1, \ldots, r$.

Then the function $f:=\left(\left.a_{i j}\right|_{U_{J}}\right)_{1 \leq 1 \leq r, 1 \leq j \leq k}: U_{J} \rightarrow M_{r k}(\mathbb{R})$ is rational and, for $S:=M_{r k}(\mathbb{Q}) \subseteq M_{r k}(\mathbb{R})$, one has $A \cap U_{J}=f^{-1}(S)$ and moreover $M_{r k}(\mathbb{R}) \backslash S$ is totally disconnected. Thus, by Lemma 3.4 exactly one of the following cases can occur:

- There exists $s \in S$ with $f(x)=s$ for all $x \in U_{J}$ (in particular $U_{J} \subseteq A$ ).

- The set $f^{-1}\left(M_{r k}(\mathbb{R}) \backslash S\right)$ is dense in $U_{J}$, and either Int $f^{-1}\left(M_{r k}(\mathbb{R}) \backslash S\right)=\emptyset$ or there exists $F_{0} \in M_{r k}(\mathbb{R}) \backslash S$ with $f\left(U_{J}\right)=\left\{F_{0}\right\}$.

Since $U_{J}$ is a dense subset of $U$ and $f^{-1}\left(M_{r k}(\mathbb{R}) \backslash S\right)=U_{J} \backslash A$, one may set $U_{0}:=U_{J}$ to complete the proof.

Lemma 3.6. Let $\mathcal{X}$ and $\mathcal{Y}$ a finite-dimensional real vector spaces and $U \subseteq \mathcal{X}$ be an open subset. Let $f_{1}, \ldots, f_{q}: U \rightarrow \mathcal{Y}$ be rational functions and define

$$
A:=\left\{x \in U \mid\left\langle f_{1}(x), \ldots, f_{q}(x)\right\rangle \text { is closed in } \mathcal{Y}\right\} \text {. }
$$

Then exactly one of the following assertions holds:

(i) The set Int $A$ is a dense open subset of $U$.

(ii) The set $U \backslash A$ is dense in $U$, and either $\operatorname{Int}(U \backslash A)=\emptyset$ or $\operatorname{Int}(U \backslash A)$ is dense in $U$.

Proof. For every $x \in U$ we define $\mathcal{E}_{x}:=\operatorname{span}_{\mathbb{R}}\left\{f_{j}(x) \mid 1 \leq j \leq q\right\}$, and we fix a point $x_{0} \in U$ with $\operatorname{dim} \mathcal{E}_{x} \leq \operatorname{dim} \mathcal{E}_{x_{0}}$ for all $x \in U$. We denote $k:=\operatorname{dim} \mathcal{E}_{x_{0}}$, $r:=q-k$, and we label $f_{1}, \ldots, f_{q}$ as $y_{1}, \ldots, y_{k}, z_{1}, \ldots, z_{r}$, with $y_{1}\left(x_{0}\right), \ldots, y_{k}\left(x_{0}\right)$ being a basis of $\mathcal{E}_{x_{0}}$.

Since $y_{1}, \ldots, y_{k}: U \rightarrow \mathcal{Y}$ are rational functions and $y_{1}\left(x_{0}\right), \ldots, y_{k}\left(x_{0}\right)$ are linearly independent, it is straightforward to obtain a Zariski open subset $U_{0} \subseteq U$ with $x_{0} \in U_{0}$, for which $y_{1}(x), \ldots, y_{k}(x)$ are linearly independent for all $x \in U_{0}$, as in 
the proof of Lemma 3.5. Since $\operatorname{dim} \mathcal{E}_{x} \leq k$ for all $x \in U$, it then follows that $y_{1}(x), \ldots, y_{k}(x)$ is a basis of $\mathcal{E}_{x}$ for all $x \in U_{0}$. In particular, for every $x \in U_{0}$ one has

$$
z_{1}(x), \ldots, z_{r}(x) \in \mathcal{E}_{x}=\operatorname{span}_{\mathbb{R}}\left\{y_{1}(x), \ldots, y_{k}(x)\right\} \text { for all } x \in U_{0} .
$$

On the other hand, denoting

$$
A_{0}:=\left\{x \in U_{0} \mid z_{1}(x), \ldots, z_{r}(x) \in \operatorname{span}_{\mathbb{Q}}\left\{y_{1}(x), \ldots, y_{k}(x)\right\}\right\}
$$

we obtain $A_{0}=A \cap U_{0}$ by Lemma 3.2. Moreover, it follows by (3.1) along with Lemma 3.5 that there exists a dense open subset $U_{00} \subseteq U_{0}$ for which exactly one of the following cases occurs:

- One has $U_{00} \subseteq A_{0}$.

- The set $U_{0} \backslash A_{0}$ is dense in $U_{0}$, and either $\operatorname{Int}\left(U_{0} \backslash A_{0}\right)=\emptyset$ or $\operatorname{Int}\left(U_{0} \backslash A_{0}\right)$ is dense in $U_{0}$.

Since $U_{0}$ is a dense open subset of $U$, this concludes the proof.

3.2. Oblique projections and an application of Moore-Penrose inverses. We start by recalling the definition of the Moore-Penrose inverse of a bounded operator in a finite dimensional Hilbert space. (See [GvL96, subsect. 5.5.4]) for the next definition, and [BB17] for the relevance of the Moore-Penrose inverse in the context of oblique projections and more references.)

Definition 3.7. Let $\mathcal{H}$ be any finite-dimensional real Hilbert space. For every operator $A \in \mathcal{B}(\mathcal{H})$ its Moore-Penrose inverse is the operator $A^{\dagger}:=B \in \mathcal{B}(\mathcal{H})$ that is uniquely determined by the equations

$$
A B A=A, B A B=B,(A B)^{*}=A B,(B A)^{*}=B A
$$

Then $A^{\dagger}$ exists for every $A \in \mathcal{B}(\mathcal{H})$.

Definition 3.8. For any fixed integer $n \geq 1$ we define the polynomial functions $\gamma_{0}, \gamma_{1}, \ldots, \gamma_{m}: \mathcal{B}\left(\mathbb{R}^{n}, \mathbb{R}^{m}\right) \rightarrow \mathbb{R}$ by the equation

$$
(\forall t \in \mathbb{R})\left(\forall A \in \mathcal{B}\left(\mathbb{R}^{n}, \mathbb{R}^{m}\right)\right) \quad \operatorname{det}\left(\mathbf{1}+t A A^{\top}\right)=\sum_{k=0}^{m} \gamma_{k}(A) t^{k}
$$

It is easily seen that $\gamma_{0}(A)=1$ and $\gamma_{m}(A)=\operatorname{det}\left(A A^{\top}\right)$ for all $A \in \mathcal{B}\left(\mathbb{R}^{n}, \mathbb{R}^{m}\right)$. The functions $\gamma_{0}, \gamma_{1}, \ldots, \gamma_{m}$ are called the Gram coefficients; cf. [DGL05, Def. 1.2].

Lemma 3.9. For any integers $m, n \geq 1$ and $A \in \mathcal{B}\left(\mathbb{R}^{n}, \mathbb{R}^{m}\right)$ the following assertions hold:

(i) If $r \in\{1, \ldots, n\}$, one has $\operatorname{dim}(\operatorname{Ker} A)=n-r$ if and only if $\gamma_{r}(A) \neq 0$ and

$$
(\forall t \in \mathbb{R}) \quad \operatorname{det}\left(\mathbf{1}+t A A^{\top}\right)=\sum_{k=0}^{r} \gamma_{k}(A) t^{k} .
$$

(ii) If $\operatorname{dim}(\operatorname{Ker} A)=n-r$, then the orthogonal projection onto $\operatorname{Ker} A$ is given by

$$
P_{\text {Ker } A}=\mathbf{1}-\frac{1}{\gamma_{r}(A)} \sum_{k=0}^{r-1}(-1)^{r-1-k} \gamma_{k}(A)\left(A^{\top} A\right)^{r-k} .
$$

(iii) If $\operatorname{dim}(\operatorname{Ker} A)=n-r$, then the Moore-Penrose inverse of $A$ is given by

$$
\left.A^{\dagger}=\frac{1}{\gamma_{r}(A)}\left(\sum_{k=0}^{r-1}(-1)^{r-1-k} \gamma_{k}(A)\left(A^{\top} A\right)^{r-1-k}\right)\right) A^{\top} \in \mathcal{B}\left(\mathbb{R}^{m}, \mathbb{R}^{n}\right) .
$$


Proof. See [DGL05, Lemmas 1.3-1.4 and Prop. 1.6].

We recall the following definition.

Definition 3.10. If $\mathfrak{U}$ is a finite-dimensional real vector space with two subspaces $\mathfrak{U}_{1}, \mathfrak{U}_{2} \subseteq \mathfrak{U}$ with $\mathfrak{U}=\mathfrak{U}_{1} \dot{+} \mathfrak{U}_{2}$, then the corresponding linear oblique projection of $\mathfrak{U}$ onto $\mathfrak{U}_{2}$ along $\mathfrak{U}_{1}$ is the linear operator $E_{\mathfrak{U}_{2}}: \mathfrak{U} \rightarrow \mathfrak{U}$ defined by the conditions $\operatorname{Ker} E_{\mathfrak{U}_{2}}=\mathfrak{U}_{1}$ and $E_{\mathfrak{U}_{2}} w=w$ for every $w \in \mathfrak{U}_{2}$.

Proposition 3.11. Let $\mathfrak{U}, \mathcal{X}$, and $\mathcal{Y}$ be finite-dimensional real vector spaces. If $U \subseteq \mathcal{X}, \mathfrak{U}_{0} \in \operatorname{Gr}(\mathfrak{U})$, and $T: U \rightarrow \mathcal{B}(\mathfrak{U}, \mathcal{Y})$ is a rational mapping with $\operatorname{Ker} T(x) \in$ $\operatorname{Gr}_{\mathfrak{U}_{0}}(\mathfrak{U})$ for all $x \in U$, then the mapping $\theta: U \rightarrow \mathcal{B}\left(\mathfrak{U}, \mathfrak{U}_{0}\right), \theta(x):=E_{\mathfrak{U}_{0}}(\operatorname{Ker} T(x))$ is rational.

Proof. Fix a scalar product on $\mathfrak{U}$, so that $\mathfrak{U}$ becomes a real Hilbert space. It follows by [BB17, Lemma 2.3] that

$$
(\forall x \in U) \quad \theta(x)=P_{\operatorname{Ker} T(x)}\left(\left(\mathbf{1}-P_{\mathfrak{U}_{0}}\right) P_{\operatorname{Ker} T(x)}\right)^{\dagger}\left(\mathbf{1}-P_{\mathfrak{U}_{0}}\right) .
$$

On the other hand, denoting $r:=\operatorname{dim} \mathfrak{U}_{0}$, for all $x \in U$ one has by hypothesis $\operatorname{Ker} T(x) \in \operatorname{Gr}_{\mathfrak{U}_{0}}(\mathfrak{U})$ hence $\operatorname{dim}(\operatorname{Ker} T(x))=\operatorname{dim} \mathfrak{U}-r$. It is also easily checked that

$$
(\forall x \in U) \quad \operatorname{Ker}\left(\left(\mathbf{1}-P_{\operatorname{Ker} T(x)}\right) P_{\mathfrak{U}_{0}}\right)=\mathfrak{U}_{0}^{\perp} .
$$

It then follows by Lemma 3.9 along with the above fomula that $\theta$ is a composition of rational mappings, hence $\theta$ is in turn rational a rational function.

The following example is needed in the proof of Corollary 4.11

Example 3.12 (Stratification according to a unipotent representation). Let $\mathfrak{g}$ be a nilpotent Lie algebra with $m:=\operatorname{dim} \mathfrak{g}$ and its corresponding group $G=(\mathfrak{g}, \cdot), \mathcal{V}$ be a finite-dimensional real vector space with $n:=\operatorname{dim} \mathcal{V}$, and $\rho: G \rightarrow \operatorname{End}(\mathcal{V})$ be a unipotent representation, that is, $(\mathbf{1}-\rho(x))^{n}=0$ for all $x \in G$. The contragredient representation $\rho^{*}: G \rightarrow \operatorname{End}\left(\mathcal{V}^{*}\right), \rho^{*}(x):=\rho(-x)^{*}$, is unipotent as well. For a Jordan-Hölder sequence of ideals of $\mathfrak{g}$,

$$
\mathfrak{F}_{\bullet}: \quad\{0\}=\mathfrak{F}_{0} \subseteq \mathfrak{F}_{1} \subseteq \cdots \subseteq \mathfrak{F}_{m}=\mathfrak{g}
$$

and a sequence of linear subspaces

$$
\mathcal{Y}_{\bullet}: \quad\{0\}=\mathcal{Y}_{0} \subseteq \mathcal{Y}_{1} \subseteq \cdots \subseteq \mathcal{Y}_{n}=\mathcal{V}^{*}
$$

with $\operatorname{dim} \mathcal{Y}_{j}=j$ and $\mathrm{d} \rho^{*}(\mathfrak{g}) \mathcal{Y}_{j} \subseteq \mathcal{Y}_{j-1}$ for $j=1, \ldots, n$, and a linear subspace $\mathfrak{h} \subseteq \mathfrak{g}$ we denote

$$
\text { jump }_{\mathfrak{F} \bullet}(\mathfrak{h}):=\left\{j \in\{1, \ldots, m\} \mid \mathfrak{F}_{j-1}+\mathfrak{h} \varsubsetneqq \mathfrak{F}_{j}+\mathfrak{h}\right\} .
$$

By the construction of [Pe94, §1.4] we obtain a stratification of $\left(\mathcal{V}^{*}\right)^{*}=\mathcal{V}$ according to the representation $\rho^{*}$. That is, we obtain a partition

$$
\mathcal{V}=\bigsqcup_{\varepsilon \in \mathcal{E}} \Omega_{\varepsilon}
$$

where the set $\mathcal{E}$ is finite and is endowed with a total ordering $\prec$ satisfying the following conditions:

(i) For every $\varepsilon \in \mathcal{E}$ the set $\Omega_{\varepsilon}$ is $\rho(G)$-invariant and there exists a subset $e(\varepsilon) \subseteq$ $\{1, \ldots, m\}$ with jump $\mathfrak{F}_{\mathfrak{\bullet}}(\mathfrak{g}(v))=e(\varepsilon)$ for all $v \in \Omega_{\varepsilon}$, where $\mathfrak{g}(v)$ is the isotropy subalgebra for $\rho$ at $v$, that is, the Lie algebra of $G(v)$. 
(ii) There exists a family of polynomial functions $\left\{Q_{\varepsilon}: \mathcal{V} \rightarrow \mathbb{R} \mid \varepsilon \in \mathcal{E}\right\}$ with $\Omega_{\varepsilon}=\left\{v \in \mathcal{V} \mid Q_{\varepsilon}(v) \neq 0=Q_{\varepsilon^{\prime}}\right.$ if $\left.\varepsilon^{\prime} \prec \varepsilon\right\}$. (See [Pe94, Prop. 1.3.2].) In particular, if $\varepsilon_{0}=\min \mathcal{E}$, then $\Omega_{\varepsilon_{0}}$ is a dense open subset of $\mathcal{V}$.

In this setting we also recall for later use that for any selection $x_{j} \in \mathfrak{g}_{j} \backslash \mathfrak{g}_{j-1}$ for $j=1, \ldots, m$,

$$
\text { if } e:=\operatorname{jump}_{\mathfrak{F}}(\mathfrak{h}) \text { and } \mathfrak{g}_{e, \mathfrak{h}}:=\operatorname{span}\left\{x_{j} \mid j \in e\right\} \text {, then } \mathfrak{h} \in \operatorname{Gr}_{\mathfrak{g} e, \mathfrak{h}}(\mathfrak{g}) \text {, }
$$

that is, there is the direct sum decomposition $\mathfrak{g}=\mathfrak{h} \dot{+} \mathfrak{g}_{e, \mathfrak{h}}$. (See BB17, Prop. $3.4(\mathrm{x})]$.) We note that actually

$$
\mathfrak{F}_{j}=\left(\mathfrak{h} \cap \mathfrak{F}_{j}\right) \dot{+}\left(\mathfrak{g}_{e, \mathfrak{h}} \cap \mathfrak{F}_{j}\right) \text { for } j=1, \ldots, m .
$$

To see this, let $q:=E_{\mathfrak{h}, \mathfrak{g}}\left(\mathfrak{g}_{e, \mathfrak{h}}\right): \mathfrak{g} \rightarrow \mathfrak{g}$ be the oblique projection onto $\mathfrak{h}$ along $\mathfrak{g}_{e, \mathfrak{h}}$ given by the direct sum decomposition $\mathfrak{g}=\mathfrak{h} \dot{+} \mathfrak{g}_{e, \mathfrak{h}}$. It suffices to show that $q\left(\mathfrak{F}_{j}\right) \subseteq \mathfrak{F}_{j}$ for $j=1, \ldots, n$. If $j=1$, then one has either $1 \in e$ and then $q\left(x_{1}\right)=$ $0 \in \mathfrak{F}_{1}$, or $1 \notin e=\operatorname{jump}_{\mathfrak{F} \bullet}(\mathfrak{h})$ and then $\mathfrak{F}_{1} \subseteq \mathfrak{h}$ hence $q\left(x_{1}\right)=x_{1} \in \mathfrak{F}_{1}$. In any of these cases we obtain $q\left(\mathfrak{F}_{1}\right)=\mathbb{R} q\left(x_{1}\right) \subseteq \mathfrak{F}_{1}$. Now let $2 \leq j \leq m$ and assume that $q\left(\mathfrak{F}_{j-1}\right) \subseteq \mathfrak{F}_{j-1}$. If $j \in e$, then $x_{j} \in \mathfrak{g}_{e, \mathfrak{h}}=\operatorname{Ker} q$, hence $q\left(x_{j}\right)=0 \in \mathfrak{F}_{j}$. If $j \notin e$, then $x_{j} \in \mathfrak{F}_{j-1}+\mathfrak{h}$, hence by $\mathfrak{F}_{j-1} \subseteq \mathfrak{F}$ we directly obtain $x_{j} \in \mathfrak{F}_{j-1}+\left(\mathfrak{h} \cap \mathfrak{F}_{j}\right)$. Therefore $q\left(x_{j}\right) \in q\left(\mathfrak{F}_{j-1}\right)+\mathfrak{h} \cap \mathfrak{F}_{j} \subseteq \mathfrak{F}_{j-1}+\mathfrak{F}_{j}=\mathfrak{F}_{j}$. Using again the induction hypothesis $q\left(\mathfrak{F}_{j-1}\right) \subseteq \mathfrak{F}_{j-1}$ we then obtain $q\left(\mathfrak{F}_{j}\right) \subseteq \mathfrak{F}_{j}$, and we are done.

\section{Main Results on REgular orbits}

In this section we establish some of our main results on regular orbits of linear dynamical systems of nilpotent Lie groups. These results include a description of the points where the orbits are regular (Theorem 4.5), a dichotomy for the set of these points (Theorem 4.10), and an interpretation of that dichotomy in terms of transformation groups $C^{*}$-algebras (Corollary 4.11), which holds if and ony if the group under consideration is abelian (Proposition 4.14).

Setting 4.1. Throughout this section we keep the following hypothesis and notation.

- $G$ is a nilpotent Lie group;

- $\pi: G \rightarrow \operatorname{End}_{\mathbb{R}}(\mathcal{V})$ is a continuous representation on the finite-dimensional real vector space $\mathcal{V}$;

- $\mathcal{V}=\mathcal{V}_{1} \dot{+} \cdots \dot{+} \mathcal{V}_{m}$ is a direct sum decomposition into $\pi(G)$-invariant subspaces satisfying the condition that for every $j=1, \ldots, m$ there exists a field $\mathbb{K}_{j} \in\{\mathbb{R}, \mathbb{C}\}$ and a $\mathbb{K}_{j}$-vector space structure of $\mathcal{V}_{j}$ that agrees with its structure of a real vector subspace of $\mathcal{V}$ and there exists a function $\chi_{j}: G \rightarrow \mathbb{K}_{j}^{\times}$ for which $\left.\pi(g)\right|_{\mathcal{V}_{j}} \in \operatorname{End}_{\mathbb{K}_{j}}\left(\mathcal{V}_{j}\right)$ and $\left(\left.\pi(g)\right|_{\mathcal{V}_{j}}-\chi_{j}(g) \operatorname{id}_{\mathcal{V}_{j}}\right)^{m_{j}}=0$ for all $g \in G$, where $m_{j}:=\operatorname{dim}_{\mathbb{K}_{j}}\left(\mathcal{V}_{j}\right)$.

- For every $v \in \mathcal{V} \backslash\{0\}$ we denote by $\operatorname{supp} v$ the set $J \subseteq\{1, \ldots, m\}$ for which there exist $v_{j} \in \mathcal{V}_{j} \backslash\{0\}$ for all $j \in J$ with $v=\sum_{j \in J} v_{j}$.

Remark 4.2. The restriction of $\pi$ to its invariant subspace $\mathcal{V}_{j}$ has a joint eigenvector by the classical theorem of S. Lie BaRa86, Ch. 8, §1, Th. 1 and Cor. 2], and it then directly follows that the function $\chi_{j}: G \rightarrow \mathbb{K}_{j}^{\times}$is a continuous morphism.

It then further follows that there exist and are uniquely determined the linear functionals $\alpha_{j}, \beta_{j}: \mathfrak{g} \rightarrow \mathbb{R}$ satisfying $[\mathfrak{g}, \mathfrak{g}] \subseteq\left(\operatorname{Ker} \alpha_{j}\right) \cap\left(\operatorname{Ker} \beta_{j}\right)$ and

$$
(\forall x \in \mathfrak{g}) \quad \chi_{j}\left(\exp _{G} x\right)=\mathrm{e}^{\alpha_{j}(x)+\mathrm{i} \beta_{j}(x)} .
$$


Definition 4.3. We define the abelian Lie group $Z:=\mathbb{K}_{1}^{\times} \times \cdots \times \mathbb{K}_{m}^{\times}$and the mappings

$$
\begin{aligned}
E: G \rightarrow \operatorname{End}_{\mathbb{R}}(\mathcal{V}), & E(g):=\chi_{1}(g) \operatorname{id}_{\mathcal{V}_{1}}+\cdots+\chi_{m}(g) \operatorname{id}_{\mathcal{V}_{m}}, \\
E_{0}: G \rightarrow Z, & E_{0}(g):=\left(\chi_{1}(g), \ldots, \chi_{m}(g)\right), \\
\nu: G \rightarrow \operatorname{End}_{\mathbb{R}}(\mathcal{V}), & \nu(g):=E(g)^{-1} \pi(g) .
\end{aligned}
$$

For every $v \in \mathcal{V}$ we also define $G_{\nu}(v):=\{g \in G \mid \nu(g) v=v\}$.

Remark 4.4. We note the following properties of the objects introduced above:

(i) Both $E$ and $\nu$ are continuous representations with $[E(G), \nu(G) \cup \pi(G)]=\{0\}$ and $\pi(\cdot)=E(\cdot) \nu(\cdot)$.

(ii) For every $g \in G$ one has $\left(\nu(g)-\mathrm{id}_{\mathcal{V}}\right)^{N}=0$, where $N=\operatorname{dim} \mathcal{V}$, and this further implies that the closed subgroup $G_{\nu}(v)$ of $G$ is connected for all $v \in \mathcal{V}$. (See [CG90, Lemma 3.1.1].)

We now state the following generalization of ACO16, Th. 3.6] from abelian to general nilpotent Lie groups.

Theorem 4.5. The following assertions are equivalent for every $v \in \mathcal{V} \backslash\{0\}$ :

(i) The subset $\pi(G) v \subseteq \mathcal{V}$ is locally compact.

(ii) The subgroup $E_{0}(G) \subseteq Z$ is closed.

(iii) The additive subgroup $\left\langle\left\{\left.\beta_{j}\right|_{\mathfrak{g}_{\nu}(v)} \mid j \in \operatorname{supp} v\right\}\right\rangle \subseteq \mathfrak{g}_{\nu}(v)^{*}$ is discrete.

The proof requires some preparation.

Notation 4.6. We denote

$$
\begin{aligned}
X: & =\{v \in \mathcal{V} \backslash\{0\} \mid \operatorname{supp} v=\{\mathbf{1}, \ldots, m\}\} \\
& =\left\{v_{1}+\cdots+v_{m} \mid v_{j} \in \mathcal{V}_{j} \backslash\{0\} \text { for } j=1, \ldots, m\right\} .
\end{aligned}
$$

For $j=1, \ldots, m$ and $v \in \mathcal{V}_{j} \backslash\{0\}$ we also denote $[v]:=\mathbb{K}_{j} v \subseteq \mathcal{V}$. We also define the projective space $\mathbb{P}\left(\mathcal{V}_{j}\right):=\left\{[v] \mid v \in \mathcal{V}_{j} \backslash\{0\}\right\}$, endowed with its usual topology of a compact space for which the mapping $\mathcal{V}_{j} \backslash\{0\} \rightarrow \mathbb{P}\left(\mathcal{V}_{j}\right), v \mapsto[v]$, is a quotient mapping.

We denote $W:=\mathbb{P}\left(\mathcal{V}_{1}\right) \times \cdots \times \mathbb{P}\left(\mathcal{V}_{m}\right)$ and set

$$
q: X \rightarrow W, \quad q\left(v_{1}+\cdots+v_{m}\right):=\left(\left[v_{1}\right], \ldots,\left[v_{m}\right]\right) .
$$

Consider the group action

$$
\widetilde{\nu}: G \times W \rightarrow W, \quad \widetilde{\nu}(g, w):=g \cdot w
$$

where $g .\left(\left[v_{1}\right], \ldots,\left[v_{m}\right]\right):=\left(\left[\nu(g) v_{1}\right], \ldots,\left[\nu(g) v_{m}\right]\right)$ for all $w=\left(\left[v_{1}\right], \ldots,\left[v_{m}\right]\right) \in W$ and $g \in G$. We note for later reference that

$$
(\forall j \in\{1, \ldots, m\})\left(\forall v_{j} \in \mathcal{V}_{j} \backslash\{0\}\right)(\forall g \in G) \quad\left[\pi(g) v_{j}\right]=\left[\nu(g) v_{j}\right] \in \mathbb{P}\left(\mathcal{V}_{j}\right) .
$$

Lemma 4.7. One has the commutative diagram

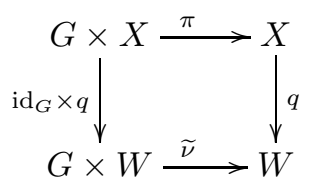

and for every $v \in X$ the mapping $\left.q\right|_{\nu(G) v}: \nu(G) v \rightarrow G \cdot q(v)=\widetilde{\nu}(G \times\{q(v)\})$ is a $G$-equivariant homeomorphism. 
Proof. Commutativity of this diagram follows by (4.1).

Now let $v=v_{1}+\cdots+v_{m} \in X$ arbitrary, where $v_{j} \in \mathcal{V}_{j} \backslash\{0\}$ for $j=1, \ldots, m$. It is easily seen that the mapping $\left.q\right|_{\nu(G) v}: \nu(G) v \rightarrow G . q(v)$ is continuous, surjective, and $G$-equivariant. To show that it is also injective, let $g_{1}, g_{2} \in G$ arbitrary with $q\left(\nu\left(g_{1}\right) v\right)=q\left(\nu\left(g_{2}\right) v\right)$. Then, for $j=1, \ldots, m$, there exists $t_{j} \in \mathbb{K}_{j}^{\times}$with $\nu\left(g_{1}\right) v_{j}=$ $t_{j} \nu\left(g_{2}\right) v_{j}$, hence $\nu\left(g_{2}^{-1} g_{1}\right) v_{j}=t_{j} v_{j}$. Now $t_{j}=1$ by Remark 4.4(iii), and then $\nu\left(g_{1}\right) v=\nu\left(g_{2}\right) v$, which shows that $\left.q\right|_{\nu(G) v}$ is injective.

It remains to check that the inverse of $\left.q\right|_{\nu(G) v}: \nu(G) v \rightarrow G . q(v)$ is continuous. To this end it suffices to prove that for every sequence $\left\{g_{n}\right\}_{n \in \mathbb{N}}$ in $G$ with $\lim _{n \rightarrow \infty} \widetilde{\nu}\left(g_{n}, q(v)\right)=q(v)$, one has $\lim _{n \rightarrow \infty} \nu\left(g_{n}\right) v=v$. In fact, for $j=1, \ldots, n$ one has $\lim _{n \rightarrow \infty}\left[\nu\left(g_{n}\right) v_{j}\right]=\left[v_{j}\right]$ in $\mathbb{P}\left(\mathcal{V}_{j}\right)$, hence there exists a sequence $\left\{t_{n}\right\}_{n \in \mathbb{N}}$ in $\mathbb{K}_{j}$ with

$$
\lim _{n \rightarrow \infty} t_{n} \nu\left(g_{n}\right) v_{j}=v_{j}
$$

in $\mathcal{V}_{j} \backslash\{0\}$. On the other hand, by S. Lie's theorem for the unipotent representation $G \rightarrow \operatorname{End}\left(\mathcal{V}_{j}\right),\left.g \mapsto \nu(g)\right|_{\mathcal{V}_{j}}$, there exists a basis in the $\mathbb{K}_{j}$-vector space $\mathcal{V}_{j}$ with respect to which one has a lower-triangular matrix representation

$$
\left.\nu(\cdot)\right|_{\mathcal{V}_{j}}=\left(\begin{array}{ccc}
1 & & 0 \\
& \ddots & \\
* & & 1
\end{array}\right)
$$

and it then easily follows by (4.2) that $\lim _{n \rightarrow \infty} t_{n}=1$ in $\mathbb{K}_{j}^{\times}$, hence one obtains by (4.2) again that $\lim _{n \rightarrow \infty} \nu\left(g_{n}\right) v_{j}=v_{j}$ for every $j$. This further implies $\lim _{n \rightarrow \infty} \nu\left(g_{n}\right) v=v$, which completes the proof of the fact that the inverse of $\left.q\right|_{\nu(G) v}: \nu(G) v \rightarrow G . q(v)$ is continuous. This concludes the proof.

Lemma 4.8. For any connected closed subgroup $H \subseteq G$ with its Lie algebra $\mathfrak{h} \subseteq \mathfrak{g}$ define $B_{H}:=\left\{\left.\beta_{j}\right|_{\mathfrak{h}} \mid j=1, \ldots, m\right\} \subseteq \mathfrak{h}^{*}$. The following conditions are equivalent:

(i) The subgroup $E_{0}(H) \subseteq Z$ is closed.

(ii) One has $\operatorname{dim}_{\mathbb{R}}\left(\operatorname{span}_{\mathbb{R}}\left(B_{H}\right)\right)=\operatorname{dim}_{\mathbb{Q}}\left(\operatorname{span}_{\mathbb{Q}}\left(B_{H}\right)\right)$.

(iii) The additive subgroup $\left\langle B_{H}\right\rangle \subseteq \mathfrak{h}^{*}$ is discrete.

Proof. It is easily seen that Assertions (iii)-(iii) are equivalent by Lemma 3.2

Let $(H, H)$ denote the subgroup of $H$ generated by its subset $\left\{h g h^{-1} g^{-1} \mid h, g \in\right.$ $H$ \}. Since $H$ is a nilpotent Lie group, $(H, H)$ is the closed connected subgroup of $H$ whose Lie algebra is $[\mathfrak{h}, \mathfrak{h}]$. (See [Ho65, Ch. XII, Th. 3.1].) Moreover, $[\mathfrak{h}, \mathfrak{h}] \subseteq \operatorname{Ker} \beta_{j}$ for $j=1, \ldots, m$, and on the other hand $(H, H) \subseteq \operatorname{Ker} E_{0}$ since $E_{0}: G \rightarrow Z$ and the group $Z$ is abelian.

Now define the abelian Lie group $\widetilde{H}:=H /(H, H)$ with its Lie algebra $\widetilde{\mathfrak{h}}:=$ $\mathfrak{h} /[\mathfrak{h}, \mathfrak{h}]$, and denote by $P: H \rightarrow \widetilde{H}$ and $p: \mathfrak{h} \rightarrow \widetilde{\mathfrak{h}}$ their corresponding quotient maps. It follows by the above observations that there exist and are uniquely determined the continuous group morphisms $\widetilde{\chi}_{j}: \widetilde{H} \rightarrow \mathbb{K}_{j}^{\times}$and linear functionals $\widetilde{\beta}_{j}: \widetilde{\mathfrak{h}} \rightarrow \mathbb{R}$ satisfying $\tilde{\chi}_{j} \circ P=\left.\chi_{j}\right|_{H}$ and $\widetilde{\beta}_{j} \circ p=\left.\beta_{j}\right|_{\mathfrak{h}}$ for $j=1, \ldots, m$. Defining $\widetilde{E}_{0}:=$ $\left(\widetilde{\chi}_{1}, \ldots, \widetilde{\chi}_{m}\right): \widetilde{H} \rightarrow Z$, one also has $\widetilde{E}_{0} \circ P=E$. If we also denote

$$
\widetilde{B_{H}}:=\left\{\widetilde{\beta}_{j} \mid j=1, \ldots, m\right\} \subseteq \widetilde{\mathfrak{h}}^{*}
$$

then Assertions (ii), (iii), and (iii) from the statement are respectively equivalent to the following assertions: 
(i') The subgroup $\widetilde{E}_{0}(\widetilde{H}) \subseteq Z$ is closed.

(ii') One has $\operatorname{dim}_{\mathbb{R}}\left(\operatorname{span}_{\mathbb{R}}\left(\widetilde{\widetilde{B_{H}}}\right)\right)=\operatorname{dim}_{\mathbb{Q}}\left(\operatorname{span}_{\mathbb{Q}}\left(\widetilde{B_{H}}\right)\right)$.

(iii') The additive subgroup $\left\langle\widetilde{B_{H}}\right\rangle=\mathbb{Z} \widetilde{\beta}_{1}+\cdots+\mathbb{Z} \widetilde{\beta}_{m} \subseteq \widetilde{\mathfrak{h}}^{*}$ is discrete.

Here $\widetilde{H}$ is an abelian (simply connected) Lie group, hence one easily obtains that Assertions (ii)-(iii) are equivalent by [Bo74, Ch. VII, §1, no. 5]. Moreover, as we already noted at the beginning of the present proof, Assertions (iii)-(iii) are equivalent by Lemma 3.2

Therefore Assertions (ii)-(iii) are equivalent.

Proof of Theorem 4.5. Let $v \in \mathcal{V} \backslash\{0\}$. Restricting the representation $\pi: G \rightarrow$ $\operatorname{End}_{\mathbb{R}}(\mathcal{V})$ to its invariant subspace $\bigoplus_{j \in \operatorname{supp} v} \mathcal{V}_{j}$, we may assume $v \in X$, hence $v=$ $v_{1}+\cdots+v_{m} \in X$ with $v_{j} \in \mathcal{V}_{j} \backslash\{0\}$ for $j=1, \ldots, m$. Denote $w:=q(v) \in W$ and its corresponding isotropy group $G_{\widetilde{\nu}}(w)=\{g \in G \mid \widetilde{\nu}(g, w)=w\}$. We actually have that

$$
G_{\widetilde{\nu}}(w)=G_{\nu}(v) .
$$

In fact, for arbitrary $g \in G$,

$$
\nu(g) v=v \Longleftrightarrow q(\nu(g) v)=q(v) \Longleftrightarrow \widetilde{\nu}(g, q(v))=q(v) \Longleftrightarrow g \in G_{\widetilde{\nu}}(w)
$$

since the mapping $\left.q\right|_{\nu(G) v}$ is injective by Lemma 4.7 and that the diagram from that lemma is commutative.

Recall from Remark 4.4(iii) that $\nu: G \rightarrow \operatorname{End}(\mathcal{V})$ is a unipotent representation, hence $\nu(G) v$ is a locally compact subset of $\mathcal{V}$. (See for instance [CG90, Th. 3.1.4].) This implies by Lemma 4.7 that the subset $G . w \subseteq W$ is locally compact. Using (4.3) and the commutative diagram from Lemma 4.7, it then follows by Lemma 2.5(iv) that one has

$$
\pi(G) v \text { locally compact } \subseteq \mathcal{V} \Longleftrightarrow \pi\left(G_{\nu}(v)\right) v \text { locally compact } \subseteq \mathcal{V} .
$$

Thus, to complete the proof that Assertions (ii)-(ii) are equivalent, it suffices to show the following:

$$
\pi\left(G_{\nu}(v)\right) v \text { locally compact } \subseteq \mathcal{V} \Longleftrightarrow E_{0}(G) \text { closed } \subseteq Z .
$$

To this end we recall that

$$
G_{\nu}(v)=\{g \in G \mid \nu(g) v=v\}=\left\{g \in G \mid \nu(g) v_{j}=v_{j} \text { for } j=1, \ldots, m\right\}
$$

hence

$$
\left(\forall g \in G_{\nu}(v)\right) \quad \pi(g) v=E(g) \nu(g) v=\sum_{j=1}^{m} E(g) \nu(g) v_{j}=\sum_{j=1}^{m} E(g) v_{j}=\sum_{j=1}^{m} \chi_{j}(g) v_{j} .
$$

This easily implies that the mapping

$$
\pi\left(G_{\nu}(v)\right) v \rightarrow E_{0}\left(G_{\nu}(v)\right), \quad \pi(g) v \mapsto\left(\chi_{1}(g), \ldots, \chi_{m}(g)\right)=E_{0}(g)
$$

is a homeomorphism, and then $\pi\left(G_{\nu}(v)\right) v$ is a locally compact subset of $\mathcal{V}$ if and only if $E_{0}\left(G_{\nu}(v)\right)$ is a locally compact subset of $Z$. This is further equivalent to the condition that $E_{0}\left(G_{\nu}(v)\right)$ be a locally closed subset of $Z$ by Wi07, Lemma 1.26]. Since $E_{0}\left(G_{\nu}(v)\right)$ is actually a subgroup of $Z$, and any subgroup of a topological group is locally closed if and only if it is closed (by [Ho65, Ch. I, Prop. 2.1]), the proof of (4.4) is complete.

Assertions (iii)-(iii) are equivalent by Lemma 4.8, This completes the proof. 
Theorem 4.10 below is a generalization of [ACO16, Cor. 5.6] from abelian to general nilpotent Lie groups. We use the following notation.

Notation 4.9. Let $k_{0}:=\min _{v \in \mathcal{V}} \operatorname{dim} \mathfrak{g}_{\nu}(v), \mathcal{V}_{\text {gen }}:=\left\{v \in \mathcal{V} \mid \operatorname{dim} \mathfrak{g}_{\nu}(v)=k_{0}\right\}$. Then the set $\mathcal{V}_{\text {gen }}$ is an open dense $\pi(G)$-invariant subset of $\mathcal{V}$ and one has $\mathcal{V}_{\text {gen }} \subseteq X$. We also consider the set

$$
\Gamma:=\{v \in \mathcal{V} \mid \pi(G) v \text { locally compact } \subseteq \mathcal{V}\}
$$

of the regular points in $\mathcal{V}$.

Theorem 4.10. Let $G$ be a nilpotent Lie group and $\pi: G \rightarrow \operatorname{End}_{\mathbb{R}}(\mathcal{V})$ a continuous representation on the finite-dimensional real vector space $\mathcal{V}$. If $\mathfrak{k} \subseteq \mathfrak{g}$ is a linear subspace for which the set

$$
\mathcal{V}_{\mathfrak{k}}:=\left\{v \in \mathcal{V} \mid \mathfrak{g}_{\nu}(v) \dot{+} \mathfrak{k}=\mathfrak{g}\right\}
$$

is a dense open subset of $\mathcal{V}_{\text {gen }}$ then exactly one of the following cases occurs:

(i) The set $\operatorname{Int}\left(\mathcal{V}_{\mathfrak{k}} \cap \Gamma\right)$ is a dense open subset of $\mathcal{V}_{\mathfrak{k}}$.

(ii) The set $\mathcal{V}_{\mathfrak{k}} \backslash \Gamma$ is dense in $\mathcal{V}_{\mathfrak{k}}$, and either $\operatorname{Int}\left(\mathcal{V}_{\mathfrak{k}} \backslash \Gamma\right)=\emptyset$ or $\operatorname{Int}\left(\mathcal{V}_{\mathfrak{k}} \backslash \Gamma\right)$ is dense in $\mathcal{V}_{\mathfrak{k}}$.

Proof. If $\mathcal{V}_{\mathfrak{k}} \cap \Gamma=\emptyset$, then (ii) clearly holds true.

Now let us assume $\mathcal{V}_{\mathfrak{k}} \cap \Gamma \neq \emptyset$ and select $v_{0} \in \mathcal{V}_{\mathfrak{k}} \cap \Gamma$. The proof proceeds in several steps. Recall that for every linear subspace $\mathfrak{h} \in \operatorname{Gr}_{\mathfrak{k}}(\mathfrak{g})$ we consider $E_{\mathfrak{k}}(\mathfrak{h}): \mathfrak{g} \rightarrow \mathfrak{g}$ its corresponding oblique projection onto $\mathfrak{h}$, corresponding to the direct sum decomposition $\mathfrak{g}=\mathfrak{h} \dot{+} \mathfrak{k}$.

Step 1. The function $\Psi: \mathcal{V}_{\mathfrak{k}} \rightarrow \mathcal{B}(\mathfrak{g}), \Psi(v):=E_{\mathfrak{k}}\left(\mathfrak{g}_{\nu}(v)\right)$ is rational.

This follows by Proposition 3.11, for $T: \mathcal{V}_{\mathfrak{k}} \rightarrow \mathcal{B}(\mathfrak{g}, \mathcal{V}), T(v):=\mathrm{d} \nu(\cdot) v$, which satisfies $\operatorname{Ker} T(v)=\mathfrak{g}_{\nu}(v) \in \operatorname{Gr}_{\mathfrak{k}}(\mathfrak{g})$ for all $v \in \mathcal{V}_{\mathfrak{k}}$.

Step 2. For every $j=1, \ldots, m$ the function

$$
f_{j}: \mathcal{V}_{\mathfrak{k}} \rightarrow \mathfrak{g}_{\nu}\left(v_{0}\right)^{*}, \quad f_{j}(v):=\left.\beta_{j} \circ \Psi(v)\right|_{\mathfrak{g}_{\nu}\left(v_{0}\right)}
$$

is rational and one has

$$
\mathcal{V}_{\mathfrak{k}} \cap \Gamma=\left\{v \in \mathcal{V}_{\mathfrak{k}} \mid\left\langle f_{1}(v), \ldots, f_{m}(v)\right\rangle \text { discrete } \subseteq \mathfrak{g}_{\nu}\left(v_{0}\right)^{*}\right\} .
$$

In fact, it directly follows by Step 1 that the functions $f_{1}, \ldots, f_{m}: \mathcal{V}_{\mathfrak{k}} \rightarrow \mathfrak{g}_{\nu}\left(v_{0}\right)^{*}$ are rational. Moreover, for arbitrary $v \in \mathcal{V}_{\text {gen }}$ one has $\mathfrak{k} \cap \mathfrak{g}_{\nu}\left(v_{0}\right)=\{0\}$. It is then straightforward to check that the linear operator

$$
\left.\Psi(v)\right|_{\mathfrak{g}_{\nu}\left(v_{0}\right)}: \mathfrak{g}_{\nu}\left(v_{0}\right) \rightarrow \mathfrak{g}_{\nu}(v)
$$

is injective hence invertible since $\operatorname{dim} \mathfrak{g}_{\nu}(v)=\operatorname{dim} \mathfrak{g}_{\nu}\left(v_{0}\right)$. One then obtains for arbitrary $v \in \mathcal{V}_{\mathfrak{k}}$

$$
\begin{aligned}
\left\langle\left.\beta_{1}\right|_{\mathfrak{g}_{\nu}(v)}, \ldots,\left.\beta_{m}\right|_{\mathfrak{g}_{\nu}(v)}\right\rangle \text { discrete } \subseteq \mathfrak{g}_{\nu}(v)^{*} & \Longleftrightarrow\left\langle\left.\beta_{1} \circ \Psi(v)\right|_{\mathfrak{g}_{\nu}\left(v_{0}\right)}, \ldots,\left.\beta_{m} \circ \Psi(v)\right|_{\mathfrak{g}_{\nu}\left(v_{0}\right)}\right\rangle \text { discrete } \subseteq \mathfrak{g}_{\nu}\left(v_{0}\right)^{*} \\
& \Longleftrightarrow\left\langle f_{1}(v), \ldots, f_{m}(v)\right\rangle \text { discrete } \subseteq \mathfrak{g}_{\nu}\left(v_{0}\right)^{*}
\end{aligned}
$$

and now (4.6) follows by Theorem 4.5 since $\mathcal{V}_{\mathfrak{k}} \subseteq \mathcal{V}_{\text {gen }} \subseteq X$. (See Notation 4.9 and 4.6.)

Step 3. The above Step 2 shows that Lemma 3.6 is applicable with $A=\mathcal{V}_{\mathfrak{k}} \cap \Gamma$, and we then obtain that either $\mathcal{V}_{\mathfrak{k}} \cap \Gamma$ is an open dense subset of $\mathcal{V}_{\mathfrak{k}}$, or $\mathcal{V}_{\mathfrak{k}} \backslash \Gamma$ $\left(=\mathcal{V}_{\mathfrak{k}} \backslash A\right)$ is a dense subset of $\mathcal{V}_{\mathfrak{k}}$. This completes the proof. 
Corollary 4.11. In the conditions and notation of Theorem 4.10, exactly one of the following cases can occur:

(i) The set Int $\Gamma$ is dense in $\mathcal{V}$.

(ii) The set $\mathcal{V} \backslash \Gamma$ is dense in $\mathcal{V}$, and either $\operatorname{Int}(\mathcal{V} \backslash \Gamma)=\emptyset$ or $\operatorname{Int}(\mathcal{V} \backslash \Gamma)$ is dense in $\mathcal{V}$.

Proof. It follows by Example 3.12(1ii) and (3.2) that there exists a linear subspace $\mathfrak{k} \subseteq \mathfrak{g}$ with $\operatorname{dim}(\mathfrak{g} / \mathfrak{k})=k_{0}$ for which the set $\mathcal{V}_{\mathfrak{k}}$ defined in (4.5) is a dense open subset of $\mathcal{V}_{\text {gen }}$. Theorem 4.10 is then applicable, and one directly obtains the assertion, since $\mathcal{V}_{\text {gen }}$ is in turn a dense open subset of $\mathcal{V}$.

Remark 4.12. The above corollary implies that $\operatorname{Int} \Gamma$ is not dense in $\mathcal{V}$ if and only if Int $\Gamma=\emptyset$.

Corollary 4.13. If the group $G$ is abelian, then exactly one of the following cases can occur:

(i) The set Int $\Gamma$ is dense in $\mathcal{V}$.

(ii) The transformation-group $C^{*}$-algebra $G \ltimes \mathcal{C}_{0}(\mathcal{V})$ is antiliminary.

Proof. The transformation-group $C^{*}$-algebra $G \ltimes \mathcal{C}_{0}(\mathcal{V})$ is not antiliminary if and only if it has at least one nonzero postliminary ideal, that is, if and only if its largest postliminary ideal is nonzero. Since the group $G$ is abelian, the largest postliminary ideal of $G \ltimes \mathcal{C}_{0}(\mathcal{V})$ is $G \ltimes \mathcal{C}_{0}(\operatorname{Int} \Gamma)$ by Lemma 2.2. Therefore $G \ltimes \mathcal{C}_{0}(\mathcal{V})$ is not antiliminary if and only if $G \ltimes \mathcal{C}_{0}(\operatorname{Int} \Gamma) \neq\{0\}$, which is further equivalent to Int $\Gamma \neq \emptyset$. Finally, by Corollary 4.11, one has $\operatorname{Int} \Gamma \neq \emptyset$ if and only if $\operatorname{Int} \Gamma$ is dense in $\mathcal{V}$.

The assertions of Corollary 4.13 do not carry over directly when $G$ is a noncommutative nilpotent Lie group, as seen from the following proposition.

Proposition 4.14. Let $G$ be a nilpotent Lie group. If $G$ is not commutative, then there exists a continuous representation $\pi: G \rightarrow \operatorname{End}_{\mathbb{C}}\left(\mathbb{C}^{2}\right)$ for which $\operatorname{Int} \Gamma=\emptyset$ while the $C^{*}$-algebra $G \ltimes_{\pi} \mathcal{C}_{0}\left(\mathbb{C}^{2}\right)$ is neither antiliminary nor postliminary.

Proof. Let $\mathfrak{g}$ be the Lie algebra of $G$ and $\mathfrak{z}$ be the center of $\mathfrak{g}$. Since $G$ is simply connected, we may assume $G=(\mathfrak{g}, \cdot)$, where the group operation $\cdot$ is given by the Baker-Campbell-Hausdorff formula. The Lie algebra $\mathfrak{g}$ is nilpotent and $\mathfrak{g}^{(1)}:=$ $[\mathfrak{g}, \mathfrak{g}] \neq\{0\}$, and it then easily follows that $\mathfrak{g}^{(1)}+\mathfrak{z} \subsetneq \mathfrak{g}$, hence we may select $X_{1} \in \mathfrak{g} \backslash\left(\mathfrak{g}^{(1)}+\mathfrak{z}\right)$, and a linear subspace $\mathfrak{g}_{0} \subseteq \mathfrak{g}$ with $\mathfrak{g}^{(1)}+\mathfrak{z} \subseteq \mathfrak{g}_{0}$ and $\mathfrak{g}=\mathbb{R} X_{1} \dot{+} \mathfrak{g}_{0}$. Then $\mathfrak{g}_{0}$ is an ideal of $\mathfrak{g}$. In addition, since $\mathfrak{z} \subseteq \mathfrak{g}_{0}$ and $X_{0} \notin \mathfrak{g}_{0}$, we have $X_{0} \notin \mathfrak{z}$, hence $\left[X_{1}, \mathfrak{g}_{0}\right] \neq\{0\}$.

Now $G_{0}:=\left(\mathfrak{g}_{0}, \cdot\right)$ is a closed normal subgroup of $G$ and we have the semidirect product decomposition $G=G_{0} \rtimes \mathbb{R} X_{1}$, using the diffeomorphism $\mathfrak{g}_{0} \times \mathbb{R} \rightarrow \mathfrak{g}$, $(Y, t) \mapsto Y \cdot t X_{1}$. We further select any $\theta \in \mathbb{R} \backslash \mathbb{Q}$ and we show that

$$
\pi: G \rightarrow \operatorname{End}_{\mathbb{C}}\left(\mathbb{C}^{2}\right), \quad \pi\left(Y \cdot t X_{1}\right):=\left(\begin{array}{cc}
\mathrm{e}^{\mathrm{i} t} & 0 \\
0 & \mathrm{e}^{\mathrm{i} \theta t}
\end{array}\right)
$$

is a representation with the stated properties. Since $G_{0}$ is a normal subgroup of $G$, it is clear that $\pi$ is a group representation. Moreover, since $\theta \in \mathbb{R} \backslash \mathbb{Q}$, it is easily 
seen that $\Gamma=\left\{\left(\begin{array}{l}z_{1} \\ z_{2}\end{array}\right) \in \mathbb{C}^{2} \mid z_{1} z_{2}=0\right\}$ hence Int $\Gamma=\emptyset$. Moreover, defining

$$
\rho: \mathbb{R} X_{1} \rightarrow \operatorname{End}_{\mathbb{R}}\left(\mathfrak{g}_{0}+\mathbb{C}^{2}\right), \quad \rho\left(t X_{1}\right):=\left(\begin{array}{cc}
\exp \left(\left.t\left(\operatorname{ad}_{\mathfrak{g}} X_{1}\right)\right|_{\mathfrak{g}_{0}}\right) & 0 \\
0 & \pi\left(t X_{1}\right)
\end{array}\right)
$$

and using the semidirect product decomposition $G=G_{0} \rtimes \mathbb{R} X_{1}$, we obtain a natural *-isomorphism $G \ltimes_{\pi} \mathcal{C}_{0}\left(\mathbb{C}^{2}\right) \simeq \mathbb{R} X_{1} \ltimes_{\rho} \mathcal{C}_{0}\left(\mathfrak{g}_{0} \dot{+} \mathbb{C}^{2}\right)$ since $G_{0}$ acts trivially on $\mathbb{C}^{2}$ via $\pi$. (See for instance Wi07, Prop. 3.11].) If we denote by $\Gamma_{\rho}$ the set of all points in $\mathfrak{g}_{0} \dot{+} \mathbb{C}^{2}$ whose corresponding orbits via the action $\rho$ are regular, then we will show that Int $\Gamma_{\rho}$ is a dense subset of $\mathfrak{g}_{0} \dot{+} \mathbb{C}^{2}$ while $\Gamma_{\rho} \neq \mathfrak{g}_{0} \dot{+} \mathbb{C}^{2}$, and this will imply that the $C^{*}$-algebra $\mathbb{R} X_{1} \ltimes_{\rho} \mathcal{C}_{0}\left(\mathfrak{g}_{0} \dot{+} \mathbb{C}^{2}\right)$ is neither postliminary (by Wi07, Thms. 6.2 and 8.43]) nor antiliminary (by Corollary 4.13).

In fact $\Gamma_{\rho} \cap \mathbb{C}^{2}=\Gamma \neq \mathbb{C}^{2}$, hence $\Gamma_{\rho} \neq \mathfrak{g}_{0} \dot{+} \mathbb{C}^{2}$. On the other hand, the nilpotent operator $\left.\left(\operatorname{ad}_{\mathfrak{g}} X_{1}\right)\right|_{\mathfrak{g}_{0}}$ is different from zero since $\left[X_{1}, \mathfrak{g}_{0}\right] \neq\{0\}$. Using the well-known formula for the exponential of a nilpotent Jordan cell, it follows that if $Y \in \mathfrak{g}_{0} \backslash \operatorname{Ker}\left(\operatorname{ad}_{\mathfrak{g}} X_{1}\right)$ and $z \in \mathbb{C}^{2}$ then $\lim _{t \rightarrow \pm \infty} \rho\left(t X_{1}\right)\left(\begin{array}{c}Y \\ z\end{array}\right)=\infty$ hence $\left(\begin{array}{c}Y \\ z\end{array}\right) \in \Gamma_{\rho}$ by the last assertion of Lemma 2.4. This shows that Int $\Gamma_{\rho}$ is a dense subset of $\mathfrak{g}_{0}+\mathbb{C}^{2}$.

Remark 4.15. As in Setting 4.1, let $G$ be a nilpotent Lie group with a continuous finite-dimensional representation $\pi: G \rightarrow \operatorname{End}_{\mathbb{R}}(\mathcal{V})$. One can then form the semidirect product of Lie groups $R:=\mathcal{V} \rtimes_{\pi} G$, which is a solvable Lie group with its Lie algebra $\mathfrak{r}:=\mathcal{V} \rtimes_{\mathrm{d} \pi} \mathfrak{g}$ obtained as a semidirect product of Lie algebras, however the relation between the coadjoint orbits of $S$ and the $G$-orbits in $\mathcal{V}$ is rather involved especially if the group $G$ is nonabelian, as discussed in Ra75] and Ba98. Specifically, the dual of the Lie algebra of $R$ is $\mathfrak{r}^{*}=\mathcal{V}^{*} \times \mathfrak{g}^{*}$, and the coadjoint action $\operatorname{Ad}_{R}^{*}: R \times \mathfrak{r}^{*} \rightarrow \mathfrak{r}^{*}$ is given by the formula

$$
\left.\operatorname{Ad}_{R}^{*}(v, g)\right)(p, \xi)=\left(\pi(g)^{*} p, \operatorname{Ad}_{G}^{*}(g) \xi-\theta_{p}(v)\right)
$$

for all $v \in \mathcal{V}, g \in G, p \in \mathcal{V}^{*}$, and $\xi \in \mathfrak{g}^{*}$, where $\theta_{p}: \mathcal{V} \rightarrow \mathfrak{g}^{*}, v \mapsto\left\langle\mathrm{d} \pi(\cdot)^{*} p, v\right\rangle$ and $\langle\cdot, \cdot\rangle: \mathcal{V}^{*} \times \mathcal{V} \rightarrow \mathbb{R}$ is the natural duality pairing.

Using these remarks, a version of Corollary 4.11 in the special case when $G$ is abelian studied in ACDO13] and ACO16] can be alternatively obtained as follows. Namely, if $G$ is abelian, then $\operatorname{Ad}_{G}^{*}(g) \xi=\xi$ for all $g \in G$ and $\xi \in \mathfrak{g}^{*}$, hence, by (4.7),

$$
\left.\operatorname{Ad}_{R}^{*}(R)\right)(p, \xi)=\pi(G)^{*} p \times\left(\xi+\theta_{p}(\mathcal{V})\right) \subseteq \mathcal{V}^{*} \times \mathfrak{g}^{*} \text { for all } p \in \mathcal{V}^{*}, \xi \in \mathfrak{g}^{*}
$$

Here $\xi+\theta_{p}(\mathcal{V})$ is an affine subspace, hence a closed subset of $\mathfrak{g}^{*}$. On the other hand, it is straightforward to check that for any topological spaces $X$ and $Y$, a subset $A \subseteq X$ is locally closed if and only if $A \times Y \subseteq X \times Y$ is locally closed. Therefore (4.8) implies that, for any $p \in \mathcal{V}^{*}$ and $\xi \in \mathfrak{g}^{*}$, the $G$-orbit $\pi(G)^{*} p \subseteq \mathcal{V}^{*}$ is locally closed if and only if the coadjoint $S$-orbit $\left(\operatorname{Ad}_{R}^{*}(R)\right)(p, \xi) \subseteq \mathfrak{r}^{*}$ is locally closed. On the other hand, since $R$ is a connected solvable Lie group, it follows by [Pu71, Ch. IV, Prop. 8.2] that, denoting by $E_{c}$ the set of all points of $\mathfrak{r}^{*}$ whose coadjoint orbits are locally closed, then either $E_{c}$ or its complement $\mathfrak{r}^{*} \backslash E_{c}$ have Lebesgue measure zero. Therefore, by the above remarks, either the set $\Gamma^{*}$ of points in $\mathcal{V}^{*}$ whose $G$-orbits are locally closed, or its complement $\mathcal{V}^{*} \backslash \Gamma^{*}$, have Lebesgue measure zero. This is the aforementioned version of Corollary 4.11 for the representation $\pi^{*}: G \rightarrow \operatorname{End}\left(\mathcal{V}^{*}\right), g \mapsto \pi\left(g^{-1}\right)^{*}$ when $G$ is abelian. 
Moreover, by (4.8) again, all $G$-orbits in $\mathcal{V}^{*}$ are locally closed if and only if all coadjoint $R$-orbits are locally closed. If this is the case, then one also has:

1. The set $\widehat{R} \backslash \widehat{G}$ is a dense open subset of the unitary dual space $\widehat{G}$ by $\operatorname{ArKS12}$,

Lemma 4.5]. (We note that $G \simeq R / \mathcal{V}$, hence $\widehat{G}$ is always a closed subset of $\widehat{R}$.)

2. The solvable Lie group $R$ is type I by [AKKo71, Th. V.3.4], since the coadjoint $R$ orbit symplectic forms are exact, for instance by [Ba98, Prop. 4.4(1.)]. Compare [ACD12, Prop. 4.3] and Theorem 5.5(1i) below.

The following example shows that Corollary 4.13 may not carry over even in the case of a noncommutative nilpotent Lie group that acts on $\mathbb{C}^{2}$ in such a manner that its corresponding orbits are regular and the coadjoint orbits of the corresponding semidirect product group are locally closed.

Example 4.16. We consider the 3-dimensional Heisenberg group

$$
H_{3}=\left\{\left(\begin{array}{ccc}
1 & a & c \\
0 & 1 & b \\
0 & 0 & 1
\end{array}\right) \mid a, b, c \in \mathbb{R}\right\}
$$

and its representation

$$
\pi: H_{3} \rightarrow \operatorname{End}_{\mathbb{C}}\left(\mathbb{C}^{2}\right), \quad \pi\left(\begin{array}{ccc}
1 & a & c \\
0 & 1 & b \\
0 & 0 & 1
\end{array}\right)=\left(\begin{array}{cc}
\mathrm{e}^{\mathrm{i} a} & 0 \\
0 & \mathrm{e}^{\mathrm{i} b}
\end{array}\right) .
$$

Then the orbit of every point of $\mathbb{C}^{2}$ is a torus of dimension 0,1 , or 2 , hence $\Gamma=\mathbb{C}^{2}$. Neverteless, as we show below, the $C^{*}$-algebra $H_{3} \ltimes \mathbb{C}_{0}\left(\mathbb{C}^{2}\right)$ is antiliminary.

In fact $H_{3} \ltimes \mathbb{C}_{0}\left(\mathbb{C}^{2}\right)$ is $*$-isomorphic to the $C^{*}$-algebra of the Dixmier group $S:=\mathbb{C}^{2} \rtimes H_{3}$ of [Di61]. If $\mathfrak{s}$ is the Lie algebra of $S$, let $S(\xi)$ be the coadjoint isotropy group of any point $\xi \in \mathfrak{s}^{*}, \mathfrak{s}(\xi)$ be the Lie algebra of $S(\xi)$, and $S(\xi)_{1}$ be the connected component of $S(\xi)$ with $\mathbf{1} \in S(\xi)_{\mathbf{1}}$. Then it is known that there exists a unique Lie group morphism $\chi_{\xi}: S(\xi)_{\mathbf{1}} \rightarrow \mathbb{T}$ whose derivative at $\mathbf{1}$ is $\left.\mathrm{i} \xi\right|_{\mathfrak{s}(\xi)}$, and we introduce as in Pu71, Def. 4.1] the reduced stabilizer of $\xi$, which is the closed subgroup of $S(\xi)$ defined by

$$
\overline{S(\xi)}:=\left\{x \in S(\xi) \mid x y x^{-1} y^{-1} \in \operatorname{Ker} \chi_{\xi} \text { for all } y \in S(\xi)\right\} .
$$

We then denote

$$
E_{\infty}:=\left\{\xi \in \mathfrak{s}^{*} \mid \text { the index of the subgroup } \overline{S(\xi)} \text { of } S(x) \text { is infinite }\right\} .
$$

A well-known property of the Dixmier group is that the set $\mathfrak{s}^{*} \backslash E_{\infty}$ has its Lebesgue measure in $\mathfrak{s}^{*}$ equal to zero. See for instance [AC20, Ex. 4.7.2] for a more precise result in this connection, due to M. Duflo. It then follows by Pu71, Lemma 9.2] that if the von Neumann algebra $\mathbf{L}(S)$ generated by the regular representation of $S$ is canonically decomposed into $w^{*}$-closed ideals of types I, II, and III, then the corresponding type I component is equal to $\{0\}$. On the other hand, the von Neumann algebra generated by the regular representation of any simply connected solvable Lie group coincides with its type I or with its type II component by Pu71, Th. 5]. It then follows that the von Neumann algebra $\mathbf{L}(S)$ coincides with its type II component. Then the regular representation of $\mathbb{C}^{2} \rtimes H_{3}$ gives a faithful type II representation of $C^{*}\left(\mathbb{C}^{2} \rtimes H_{3}\right)$, and this implies that $C^{*}\left(\mathbb{C}^{2} \rtimes H_{3}\right)$ is antiliminary. (See for instance [Di69, 9.5.4].) 


\section{Applichtion to Generalized $a x+b$-Groups}

A generalized $a x+b$-group is a connected simply connected solvable Lie group having a 1-codimensional abelian ideal. See also Definition 5.4 for an alternative description of these groups. We study the type I ideals of the $C^{*}$-algebra of such a group, and our main result (Theorem [5.5) is a precise characterization of the generalized $a x+b$-groups whose $C^{*}$-algebra is antiliminary, that is, no closed 2sided ideal is type I. We also discuss the representation theoretic significance of this property (Remark 5.6).

We recall that a separable $C^{*}$-algebra $\mathcal{A}$ is type I or postliminary if for every irreducible *-representation $\pi: \mathcal{A} \rightarrow \mathcal{B}(\mathcal{H})$ one has $\mathcal{K}(\mathcal{H}) \subseteq \pi(\mathcal{A})$. The $C^{*}$-algebra $\mathcal{A}$ is called antiliminary if it has no postliminary closed 2-sided ideal different from $\{0\}$. (See Di69.)

In what follows in this section, unless otherwise mentioned, $\mathcal{V}$ is a finite-dimensional real vector space and $D \in \operatorname{End}(\mathcal{V})$. We recall the following definitions. (See [CK14, §1.4], and BB18 for the relevance of these notions in the context of $C^{*}$-algebraic properties of $a x+b$-groups.) We consider the $\mathbb{C}$-linear extension $D: \mathcal{V}_{\mathbb{C}} \rightarrow \mathcal{V}_{\mathbb{C}}$, and let $\sigma(D)$ be its spectrum. For $\lambda \in \sigma(D) \cap(\mathbb{C} \backslash \mathbb{R})$, the real generalized eigenspace for $\lambda, \bar{\lambda}$ is the linear subspace of $\mathcal{V}$ given by

$$
E^{D}(\lambda):=\left\{v_{1}, v_{2} \in \mathcal{V} \mid v_{1}+\mathrm{i} v_{2} \in \operatorname{Ker}(D-\lambda \mathbf{1})^{m}\right\},
$$

where $m$ is the dimension of the largest Jordan block for $\lambda$, while if $\lambda \in \sigma(D) \cap \mathbb{R}$, the real generalized eigenspace for $\lambda$ is $E^{D}(\lambda):=\operatorname{Ker}(D-\lambda \mathbf{1})^{m}$, where $m$ is again the dimension of the largest Jordan block for $\lambda$. For $\lambda \in \mathbb{C} \backslash \sigma(D)$, we set $E^{D}(\lambda):=\{0\}$. Then we define $\mathcal{V}_{ \pm}:=\bigoplus_{ \pm \operatorname{Re} \lambda>0} E^{D}(\lambda), \mathcal{V}_{0}:=\underset{\operatorname{Re} \lambda=0}{\bigoplus} E^{D}(\lambda)$. It follows that $\mathcal{V}=\mathcal{V}_{-} \oplus \mathcal{V}_{0} \oplus \mathcal{V}_{+}$Define

$$
D_{0}:=\left.D\right|_{\mathcal{V}_{0}} .
$$

We start with the following lemma, included here for the sake of completeness.

Lemma 5.1. For any set $S \subseteq \mathbb{R}$ the subgroup of $(\mathbb{R},+)$ generated by $S$ is closed if and only if there exists $\theta \in \mathbb{R} \backslash\{0\}$ with $S \subseteq\{r \theta \mid r \in \mathbb{Q}\}$, which is further equivalent to $\operatorname{dim}_{\mathbb{Q}}\left(\operatorname{span}_{\mathbb{Q}}(S)\right)=1$.

Proof. This follows from the well-known fact that for any $\theta_{1}, \theta_{2} \in \mathbb{R} \backslash\{0\}$ the set $\left\{m_{1} \theta_{1}+m_{2} \theta_{2} \mid m_{1}, m_{2} \in \mathbb{Z}\right\}$ is dense in $\mathbb{R}$ if and only if $\theta_{1} / \theta_{2} \in \mathbb{R} \backslash \mathbb{Q}$.

Notation 5.2. For any $D \in \operatorname{End}(\mathcal{V})$, we denote by $S_{D}:=\langle\mathrm{i} \sigma(D) \cap \mathbb{R}\rangle$ the subgroup of $(\mathbb{R},+)$ generated by the imaginary parts of the purely imaginary eigenvalues of $D$.

Some of the assertions of Lemma 5.3 below are stated without proof in ACDO13. Recall that $\Gamma$ is the set of $v \in \mathcal{V}$ for which the image of the map $\gamma_{v}: \mathbb{R} \rightarrow \mathcal{V}$, $t \mapsto \mathrm{e}^{t D} v$, is a locally closed subset of $\mathcal{V}$.

Lemma 5.3. Let $\mathcal{V}$ be a finite-dimensional real vector space, $D \in \operatorname{End}(\mathcal{V})$.

(a) The following conditions are equivalent.

(i) $\Gamma=\mathcal{V}$.

(ii) $S_{D}$ is closed.

(b) If the set $S_{D}$ is not closed, then $\operatorname{Int} \Gamma$ is not dense in $\mathcal{V}$ if and only if $\sigma(D) \subseteq$ $\mathrm{i} \mathbb{R}$ and $D$ is semisimple. 
Proof. By [BB18, Lemma 2.1 and proof of Th. 2.2] we may assume without loss of generality

$$
D=\left(\begin{array}{ccc}
-\mathrm{id}_{\mathcal{V}_{-}} & 0 & 0 \\
0 & D_{0} & 0 \\
0 & 0 & \mathrm{id}_{\mathcal{V}_{+}}
\end{array}\right)
$$

with respect to the direct sum decomposition $\mathcal{V}=\mathcal{V}_{-} \oplus \mathcal{V}_{0} \oplus \mathcal{V}_{+}$. For every $v \in \mathcal{V}$ we denote by $v=v_{-}+v_{0}+v_{+}$its decomposition with $v_{0} \in \mathcal{V}_{0}$ and $v_{ \pm} \in \mathcal{V}_{ \pm}$.

If $\mathcal{V} \neq \mathcal{V}_{0}$ and $v \in \mathcal{V} \backslash \mathcal{V}_{0}$, then either $v_{-} \neq 0$ or $v_{+} \neq 0$, hence by (5.1) the mapping $\mathbb{R} \rightarrow \mathcal{V}, t \mapsto \mathrm{e}^{t D} v$, is injective and then Lemma 2.4(iv) implies that the orbit $\mathrm{e}^{\mathbb{R} D} v$ is locally closed in $\mathcal{V}$. Indeed, if for instance $v_{-} \neq 0, \lim _{n \in \mathbb{N}}\left|t_{n}\right|=\infty$, and $\lim _{n \in \mathbb{N}} \mathrm{e}^{t_{n} D} v=v$, then one has $v_{-}=\lim _{n \in \mathbb{N}} \mathrm{e}^{t_{n} D} v_{-}=\lim _{n \in \mathbb{N}} \mathrm{e}^{t_{n}} v_{-}$. Since $v_{-} \neq 0$, this is impossible since $\lim _{n \in \mathbb{N}}\left|t_{n}\right|=\infty$ and at least one of the sets $\left\{n \in \mathbb{N} \mid t_{n}>0\right\}$ and $\left\{n \in \mathbb{N} \mid t_{n}<0\right\}$ is infinite. It follows that $\mathcal{V} \backslash \mathcal{V}_{0} \subseteq \Gamma$.

(a) (ii) $\Rightarrow$ (iii) It is enough to show that there is $\theta \in \mathbb{R}$ such that $\mathrm{i} \sigma(D) \cap \mathbb{R} \subseteq \theta \mathbb{Q}$. To this end we prove that for any $\theta_{1}, \theta_{2} \in \mathbb{R} \backslash\{0\}$ are such that $\mathrm{i} \theta_{1}, \mathrm{i} \theta_{2} \in \sigma(D) \cap \mathrm{i} \mathbb{R}$, then $\theta_{1} / \theta_{2} \in \mathbb{Q}$. We will prove this by contradiction, so let us assume $\theta_{1} / \theta_{2} \in \mathbb{R} \backslash \mathbb{Q}$.

We select $\mathbb{R}$-linearly independent $x_{1}, y_{1}, x_{2}, y_{2} \in \mathcal{V} \backslash\{0\}$ with $D\left(x_{j}+\mathrm{i} y_{j}\right)=$ $\mathrm{i} \theta_{j}\left(x_{j}+\mathrm{i} y_{j}\right) \in \mathcal{V}_{\mathbb{C}}=\mathbb{C} \otimes_{\mathbb{R}} \mathcal{V}$ for $j=1,2$, and let us define $v:=x_{1}+y_{1}+x_{2}+y_{2}$. We also denote $\mathcal{X}:=\operatorname{span}_{\mathbb{R}}\left\{x_{1}, x_{2}, y_{1}, y_{2}\right\} \subset \mathcal{V}$ and define the $\mathbb{R}$-linear isomorphism $B: \mathcal{X} \rightarrow \mathbb{C}^{2}$ with $B\left(x_{1}+y_{1}\right)=(1,0)$ and $B\left(x_{2}+y_{2}\right)=(0,1)$. Then the point $w:=(1,1) \in \mathbb{C}^{2}$ satisfies $w=B v$ and, defining $\gamma(t):=B \mathrm{e}^{t D} B^{-1}$, one has

$$
\gamma(t)=\left(\begin{array}{cc}
\mathrm{e}^{\mathrm{i} t \theta_{1}} & 0 \\
0 & \mathrm{e}^{\mathrm{i} t \theta_{2}}
\end{array}\right)
$$

Since $\theta_{1} / \theta_{2} \in \mathbb{R} \backslash \mathbb{Q}$, it is easily checked that the map $\gamma(\cdot) w$ is injective and on the other hand, it is well known that its image is dense in the torus $\mathbb{T}^{2}$. Therefore, if $\gamma(\mathbb{R}) w$ were a locally closed subset of $\mathbb{C}$, then $\gamma(\mathbb{R}) w$ would be an open subset of the torus $\mathbb{T}^{2}$, which is not the case since $\gamma(\mathbb{R})$ is a subgroup of $\mathbb{T}^{2}$ and $\gamma(\mathbb{R}) \varsubsetneqq \mathbb{T}^{2}$. It then follows that neither $B^{-1} \gamma(\mathbb{R}) w$ is a locally closed subset of $\mathcal{X}$, that is, the image of the map $\gamma_{v}$ fails to be a locally closed subset of $\mathcal{V}$, which is a contradiction with (ii).

(iii) $\Rightarrow$ (ii) It remains to show that $\mathcal{V}_{0} \subseteq \Gamma$.

Let $D_{0}=S_{0}+N_{0}$ be the Jordan decomposition with $S_{0}, N_{0} \in \operatorname{End}\left(\mathcal{V}_{0}\right)$, where $S_{0}$ is semisimple, $N_{0}$ is nilpotent, and $S_{0} N_{0}=N_{0} S_{0}$.

Specializing Setting 4.1 for $G=(\mathbb{R},+)$ and $\pi(t):=\mathrm{e}^{t S_{0}}$ for all $t \in \mathbb{R}$, we may assume that $\mathcal{V}_{0}=\mathbb{C}^{n}$ and $S_{0}$ is a diagonal matrix having purely imaginary diagonal entries, say

$$
S_{0}=\left(\begin{array}{ccc}
\mathrm{i} \theta_{1} & & 0 \\
& \ddots & \\
0 & & \mathrm{i} \theta_{n}
\end{array}\right)
$$

By the hypothesis (iii), there exist $\theta \in \mathbb{R} \backslash\{0\}$ and $p_{1}, \ldots, p_{n} \in \mathbb{Z}, q_{1}, \ldots, q_{n} \in \mathbb{N}$ with $\theta_{j}=\theta p_{j} / q_{j}$ for $j=1, \ldots, n$. Then, the group morphism $\gamma: \mathbb{R} \rightarrow U(n)\left(\subseteq M_{n}(\mathbb{C})\right)$, $\gamma(t):=\mathrm{e}^{t S_{0}}$, satisfies $\gamma\left(2 \pi q_{1} \cdots q_{n} / \theta\right)=\mathbf{1}$. Therefore $\gamma(\mathbb{R})$ is a homeomorphic image of the compact group $\mathbb{R} / \operatorname{Ker} \gamma \simeq \mathbb{R} / \mathbb{Z}$, that is, $\gamma(\mathbb{R})$ is compact. This directly implies that for every $v \in \mathcal{V}_{0}$ its orbit $\mathrm{e}^{\mathbb{R} S_{0}} v=\gamma(\mathbb{R}) v$ is a compact subset of $\mathcal{V}_{0}$, and in particular is locally closed.

When $D_{0}$ is semisimple, $D_{0}=S_{0}$, and then it follows that $\mathcal{V}_{0} \subseteq \Gamma$. 
If $D_{0}$ is not semisimple, then $N_{0} \neq 0$. For every $v \in \mathcal{V}_{0} \backslash \operatorname{Ker} N_{0}, \lim _{t \rightarrow \pm \infty}\left\|\mathrm{e}^{t D_{0}} v\right\|=$ $\infty$ (see for instance [BB18, Step 2 in the proof of Lemma 2.13]). This implies that $\lim _{t \rightarrow \pm \infty}\left\|\mathrm{e}^{t D} v\right\|=\infty$ for every $v \in \mathcal{V}$ with $v_{0} \in \mathcal{V}_{0} \backslash$ Ker $N_{0}$, hence the orbit $\mathrm{e}^{\mathbb{R} D} v$ is locally closed by Lemma $2.4((\sqrt{\mathrm{V}}) \Rightarrow(\mathrm{i}))$. On the other hand $\left.D_{0}\right|_{\operatorname{Ker} N_{0}}=\left.S_{0}\right|_{\operatorname{Ker} N_{0}}$, thus the orbit $\mathrm{e}^{\mathbb{R} D} v$ is locally closed even for $v \in \operatorname{Ker} N_{0}$, hence again $\mathcal{V}_{0} \subseteq \Gamma$.

(b) Assume now that $S_{D}$ is not a closed subgroup in $\mathbb{R}$.

If that Int $\Gamma$ is not dense in $\mathcal{V}$, then Corollary 4.11 or Remark 4.12, show that if Int $\Gamma$ is not dense in $\mathcal{V}$ then Int $\Gamma=\emptyset$. Since $\mathcal{V} \backslash \mathcal{V}_{0} \subseteq \Gamma$, it follows that $\mathcal{V}=\mathcal{V}_{0}$, or equivalently, $\sigma(D) \subset \mathrm{i} \mathbb{R}$. Then $D=D_{0}$. Assume that $D$ is not semisimple, that is, $D=D_{0}=S_{0}+N_{0}$ with $N_{0} \neq 0$, then the argument above shows that $\mathcal{V}_{0} \backslash \operatorname{Ker} N_{0} \subseteq \Gamma$, hence Int $\Gamma \neq \emptyset$. Therefore $D$ must be semisimple.

Assume now that $D$ is semisimple and $\sigma(D) \subset \mathrm{i} \mathbb{R}$. In this case, specializing Setting 4.1 for $G=(\mathbb{R},+)$ and $\pi(t):=\mathrm{e}^{t D}$ for all $t \in G$, we may assume that $\mathcal{V}=\mathbb{C}^{n}$ and $D$ is a diagonal matrix having purely imaginary diagonal entries. Then, in the notation of Definition 4.3, $G_{\nu}(v)=G=\mathbb{R}$ hence $\mathfrak{g}_{\nu}(v)=\mathbb{R}$ for every $v \in \mathcal{V}$. Then Theorem 4.5 along with the hypothesis that $S_{D}$ is not closed in $\mathbb{R}$ imply that the orbit $\mathrm{e}^{\mathbb{R} D} v$ is not locally compact in $\mathcal{V}$ for every $v \in\left(\mathbb{C}^{\times}\right)^{n}$. This shows that $\operatorname{Int} \Gamma=\emptyset$.

Definition 5.4. We define the Lie algebra $\mathfrak{g}_{D}:=\mathcal{V} \rtimes_{D} \mathbb{R}$ with its Lie bracket

$$
\left[\left(v_{1}, t_{1}\right),\left(v_{2}, t_{2}\right)\right]:=\left(t_{1} D v_{2}-t_{2} D v_{1}, 0\right)
$$

and the Lie group $G_{D}:=\mathcal{V} \rtimes_{D} \mathbb{R}$ with its product given by

$$
\left(v_{1}, t_{1}\right) \cdot\left(v_{2}, t_{2}\right)=\left(v_{1}+\mathrm{e}^{t_{1} D} v_{2}, t_{1}+t_{2}\right) .
$$

Then $\mathfrak{g}_{D}$ is the Lie algebra of $G_{D}$.

Assertion (ii) in the following theorem is well known and we give it a very short proof for the sake of completeness.

Theorem 5.5. For arbitrary $D \in \operatorname{End}(\mathcal{V})$, the following assertions holds:

(i) The set $S_{D}$ is closed in $\mathbb{R}$ if and only if $C^{*}\left(G_{D}\right)$ is type $I$.

(ii) If $S_{D}$ is not closed in $\mathbb{R}$, then $D \in \operatorname{End}(\mathcal{V})$ is semisimple and $\sigma(D) \subset \mathrm{i} \mathbb{R}$ if and only if $C^{*}\left(G_{D}\right)$ is antiliminary.

Proof. (ii) Let $\Gamma^{*}$ be the set of all $\xi \in \mathcal{V}^{*}$ whose orbit $\mathrm{e}^{\mathbb{R} D^{*}} \xi$ is locally closed in $\mathcal{V}^{*}$, and recall that $\sigma\left(D^{*}\right)=\sigma(D)$. Then, by Lemma 5.3)(a) applied for $D^{*}$ instead of $D$, the set $S_{D}$ is closed in $\mathbb{R}$ if and only if $\Gamma^{*}=\mathcal{V}^{*}$. On the other hand, since $C^{*}\left(G_{D}\right) \simeq \mathbb{R} \ltimes_{\alpha_{D^{*}}} \mathcal{C}_{0}\left(\mathcal{V}^{*}\right)$ (see Remark 2.3), it follows either by Go73, Th. 3.3] or by Lemma 2.2 that $\Gamma^{*}=\mathcal{V}^{*}$ if and only if $C^{*}\left(G_{D}\right)$ is type I.

(ii) As above, by Lemma 5.3 (b) applied for $D^{*}$ instead of $D$, if $S_{D}$ is not closed in $\mathbb{R}$ then the conditions $D \in \operatorname{End}(\mathcal{V})$ is semisimple and $\sigma(D) \subset \mathrm{i} \mathbb{R}$ are satisfied if and only if Int $\Gamma^{*}=\emptyset$. By Remark 4.12, this is the case if and only if $\operatorname{Int} \Gamma^{*}$ is not dense in $\mathcal{V}^{*}$, that is, by Corollary 4.11 if and only if $C^{*}\left(G_{D}\right)$ is antiliminary.

Remark 5.6. For any $C^{*}$-algebra $\mathcal{A}$ let us denote by $F(\mathcal{A})$ its set of factorial states, that is, the states $\varphi \in \mathcal{A}^{*}$ whose corresponding GNS representation $\pi_{\varphi}: \mathcal{A} \rightarrow \mathcal{B}\left(\mathcal{H}_{\varphi}\right)$ satisfies $\pi_{\varphi}(\mathcal{A})^{\prime} \cap \pi_{\varphi}(\mathcal{A})^{\prime \prime}=\mathbb{C} \mathbf{1}$. We also denote by $F_{\mathrm{III}}(\mathcal{A})$ the set of all $\varphi \in F(\mathcal{A})$ for which the factor $\pi_{\varphi}(\mathcal{A})^{\prime \prime}$ is type III. We endow $F(\mathcal{A})$ with its weak*-topology inherited as a subset of the dual space $\mathcal{A}^{*}$. Then the $C^{*}$-algebra $\mathcal{A}$ is antiliminary 
if and only if $F_{\mathrm{III}}(\mathcal{A})$ is dense in $F(\mathcal{A})$, by $\operatorname{ArBa86}$, Th. 2.1]. (The analogous result for type II also holds if $\mathcal{A}$ is separable.)

Let $G$ be a locally compact group. It follows from the aforementioned result along with Di69, 18.1.4] that $C^{*}(G)$ is antiliminary if and only if every factor representation of $G$ is weakly contained in the type III factor representations of $G$, in the sense that every coefficient of any factor representation of $G$ can be uniformly approximated on compacts by coefficients of type III factor representations of $G$.

On the other hand, if $G$ is a connected, simply connected, solvable Lie group, and its quasi-dual $\bar{G}$ is endowed with the equivalence class of measures arising from the factor disintegrations of left regular representation as in [Di69, 8.4.3], then the subset $\bar{G}_{\text {III }}$ of $\bar{G}$ corresponding to the type III factor representations is negligible by $\mathrm{Pu} 71$, Ch. IV, Cor. 7.2].

Nevertheless, $\bar{G}_{\text {III }}$ may not be negligible from a topological point of view. Specifically, it follows by the above remarks that Theorem 5.5 provides specific examples illustrating both cases that can occur: either $\bar{G}$ is weakly contained in (hence weakly equivalent to) $\bar{G}_{\mathrm{III}}$ or not, depending on whether $C^{*}(G)$ is antiliminary or not.

Example 5.7. Recalling the Mautner group $G_{\theta}:=G_{D_{\theta}}=\mathbb{C}^{2} \rtimes_{\alpha_{D_{\theta}}} \mathbb{R}$ defined by

$$
D_{\theta}=\left(\begin{array}{cc}
\mathrm{i} & 0 \\
0 & \mathrm{i} \theta
\end{array}\right) \in M_{2}(\mathbb{C})
$$

for $\theta \in \mathbb{R} \backslash \mathbb{Q}$, it follows by Theorem 5.5 that $C^{*}\left(G_{\theta}\right)$ is antiliminary. Also, $C^{*}\left(G_{\theta}\right)$ is not a simple $C^{*}$-algebra, in the sense that it has nontrivial closed 2 -sided ideals. For instance, using the short exact sequence of groups

$$
0 \rightarrow \mathbb{C}^{2} \hookrightarrow G_{\theta} \rightarrow \mathbb{R} \rightarrow 0
$$

one obtains the short exact sequence

$$
0 \rightarrow \mathcal{J} \hookrightarrow C^{*}\left(G_{\theta}\right) \rightarrow \mathcal{C}_{0}(\mathbb{R}) \rightarrow 0
$$

for a suitable nontrivial ideal $\mathcal{J}$. The ideal $\mathcal{J}$ is antiliminary since $C^{*}\left(G_{\theta}\right)$ is antiliminary.

On the other hand, every primitive ideal of $C^{*}\left(G_{\theta}\right)$ is maximal, by [Pu73, Th. 2]. Equivalently, for every irreducible $*$-representation $\pi: C^{*}\left(G_{\theta}\right) \rightarrow \mathcal{B}\left(\mathcal{H}_{\pi}\right)$ its corresponding primitive quotient $C^{*}\left(G_{\theta}\right) / \operatorname{Ker} \pi \simeq \pi\left(C^{*}\left(G_{\theta}\right)\right)$ is a simple $C^{*}$-algebra. All the simple $C^{*}$-algebras that arise in this way are quasidiagonal, hence $C^{*}\left(G_{\theta}\right)$ is strongly quasidiagonal (see $\mathrm{BB20}$ ). It was already known that the $C^{*}$-algebra $C^{*}\left(G_{\theta}\right)$ is quasidiagonal, as noted in [BB18, end of Sect. 2].

Acknowledgment. We wish to thank the Referees for their generous remarks and suggestions, which in particular led to Proposition 4.14 and Example 4.16.

\section{REFERENCES}

[ACM13] E. Andruchow, G. Corach, M. Mbekhta, A geometry for the set of split operators. Integral Equations Operator Theory 77 (2013), no. 4, 559-579.

[aHWi02] A. AN Huef, D.P. Williams, Ideals in transformation-group $C^{*}$-algebras. J. Operator Theory 48 (2002), no. 3, suppl., 535-548.

[ArBa86] R.J. ARchbold, C.J.K. BAtTy, On factorial states of operator algebras. III. J. Operator Theory 15 (1986), no. 1, 53-81.

[ArKS12] R.J. Archbold, E. Kaniuth, D.W.B. Somerset, Norms of inner derivations for multiplier algebras of $C^{*}$-algebras and group $C^{*}$-algebras. J. Funct. Anal. 262 (2012), no. 5, 2050-2073. 
[ArKS15] R.J. Archbold, E. Kaniuth, D.W.B. Somerset, Norms of inner derivations for multiplier algebras of $C^{*}$-algebras and group $C^{*}$-algebras, II. Adv. Math. 280 (2015), $225-255$.

[AC20] D. Arnal, B. Currey III, Representations of solvable Lie groups. Cambridge University Press, 2020.

[ACD12] D. Arnal, B. Currey, B. Dali, Canonical coordinates for a class of solvable groups. Monatsh. Math. 166 (2012), no. 1, 19-55.

[ACDO13] D. Arnal, B. Currey, B. Dali, V. Oussa, Regularity of abelian linear actions. In: A. Mayeli, A. Iosevich, P.E.T. Jorgensen, G. Ólafsson (eds.), Commutative and noncommutative harmonic analysis and applications, Contemp. Math., 603, Amer. Math. Soc., Providence, RI, 2013, pp. 89-109.

[ACO16] D. Arnal, B. Currey, V. Oussa, Characterization of regularity for a connected Abelian action. Monatsh. Math. 180 (2016), no. 1, 1-37.

[ACD19] D. Arnal, B. Currey, B. Dali, The Plancherel formula for an inhomogeneous vector group. J. Fourier Anal. Appl. 25 (2019), no. 6, 2837-2876.

[AuKo71] L. Auslander, B. Kostant, Polarization and unitary representations of solvable Lie groups. Invent. Math. 14 (1971), 255-354.

[Ba98] P. Baguis, Semidirect products and the Pukanszky condition. J. Geom. Phys. 25 (1998), no. 3-4, 245-270.

[BaRa86] A.O. BARUt, R. RA̧CZKA, Theory of group representations and applications. Second edition. World Scientific Publishing Co., Singapore, 1986.

[BB17] I. Beltiţă, D. Beltiţă, Nonlinear oblique projections. Linear Algebra Appl. 533 (2017), 451-467.

[BB18] I. Beltiţă, D. Beltiţă, Quasidiagonality of $C^{*}$-algebras of solvable Lie groups. Integral Equations Operator Theory 90 (2018), no. 1, Art. 5, 21 pp.

[BB20] I. Beltiţă, D. Beltiţă, AF-embeddability for Lie groups with $T_{1}$ primitive ideal spaces. J. London Math. Soc (to appear; see arXiv:2004.11010v3 [math.OA]).

[Bo74] N. Bourbaki, Topologie générale. Chap. 5 à 10. Nouvelle édition. Hermann, Paris, 1974.

[BCFM15] J. Bruna, J. Cufí, H. Führ, M. Miró, Characterizing abelian admissible groups. J. Geom. Anal. 25 (2015), no. 2, 1045-1074.

[Ca05] R.W. CARTER, Lie algebras of finite and affine type. Cambridge Studies in Advanced Mathematics, 96. Cambridge University Press, Cambridge, 2005.

[CK14] F. Colonius, W. Kliemann, Dynamical systems and linear algebra. Graduate Studies in Mathematics 158. American Mathematical Society, Providence, RI, 2014

[CG90] L.J. Corwin, F.P. Greenleaf, Representations of nilpotent Lie groups and their applications. Cambridge Studies in Advanced Mathematics, 18. Cambridge University Press, Cambridge, 1990.

[Di61] J. Dixmier, Sur le revêtement universel d'un groupe de Lie de type I. C. R. Acad. Sci. Paris 252 (1961), 2805-2806.

[Di69] J. Dixmier, Les $C^{*}$-algèbres et leurs représentations. Deuxième édition. Cahiers Scientifiques, Fasc. XXIX. Gauthier-Villars Éditeur, Paris, 1969

[DGL05] G.M. Diaz-Toca, L. Gonzalez-Vega, H. Lombardi, Generalizing Cramer's rule: solving uniformly linear systems of equations. SIAM J. Matrix Anal. Appl. 27 (2005), no. 3, 621-637.

[GvL96] G.H. Golub, C.F. van LoAn, Matrix computations. Third edition. Johns Hopkins Studies in the Mathematical Sciences. Johns Hopkins University Press, Baltimore, MD, 1996.

[Go73] E.C. Gootman, The type of some $C^{*}$ - and $W^{*}$-algebras associated with transformation groups. Pacific J. Math. 48 (1973), 93-106.

[Ho65] G. Hochschild, The structure of Lie groups. Holden-Day, Inc., San FranciscoLondon-Amsterdam, 1965.

[Pe94] N.V. Pedersen, Orbits and primitive ideals of solvable Lie algebras. Math. Ann. 298 (1994), no. 2, 275-326.

[Pu71] L. Pukanszky, Unitary representations of solvable Lie groups. Ann. Sci. École Norm. Sup. (4) 4 (1971), 457-608.

[Pu73] L. PukAnszky, The primitive ideal space of solvable Lie groups. Invent. Math. 22 (1973), 75-118. 
[Ra75] J.H. RAWNSLEY, Representations of a semi-direct product by quantization. Math. Proc. Cambridge Philos. Soc. 78 (1975), no. 2, 345-350.

[Wi07] D.P. Williams, Crossed products of $C^{*}$-algebras. Mathematical Surveys and Monographs, 134. American Mathematical Society, Providence, RI, 2007.

Institute of Mathematics "Simion Stollow" of the Romanian Academy, P.O. Box 1764, Bucharest, Romania

Email address: ingrid.beltita@gmail.com, Ingrid.Beltita@imar.ro

Email address: beltita@gmail.com, Daniel.Beltita@imar.ro 